\title{
Psychische Belastung und Lebensqualität bei Tinnituspatienten
}

Dissertation zur Erlangung des Grades eines Dr. med. an der Medizinischen Fakultät der Universität Leipzig

Steffi Hesse 



\title{
Psychische Belastung und Lebensqualität bei Tinnituspatienten
}

\author{
Dissertation \\ zur Erlangung des akademischen Grades \\ Dr. med. \\ an der Medizinischen Fakultät \\ der Universität Leipzig
}

eingereicht von:

Dipl.-Med. Steffi Hesse, geb. Hesse

Geburtsdatum / Geburtsort: 11.01.1964 / Leipzig

angefertigt an:

Abteilung für Medizinische Psychologie und

Medizinische Soziologie der Universität Leipzig

Betreuer:

Prof. Dr. rer. nat. habil. A. Hinz

Dr. rer. med. A. Meyer

Beschluss über die Verleihung des Doktorgrades vom: 29.04.2014 



\section{Inhaltsverzeichnis}

1. Bibliographische Zusammenfassung mit Referat 5

$\begin{array}{ll}\text { 2. Einführung } & 7\end{array}$

$\begin{array}{ll}\text { 3. Publikation } & 19\end{array}$

4. Zusammenfassung 29

5. Erklärung über die eigenständige Abfassung der Arbeit 33

6. Danksagung 35

$\begin{array}{ll}\text { 7. Lebenslauf } & 37\end{array}$ 



\title{
1. Bibliographische Beschreibung
}

\author{
Name, Vorname Hesse, Steffi \\ Titel der Arbeit

\section{Psychische Belastung und Lebensqualität bei Tinnituspatienten}

Universität Leipzig, Dissertation

8 S., 44 Lit., 1 Abb., 3 Tab.

\section{Referat:}

Tinnitus ist eine Erkrankung mit hoher Prävalenz, welche häufig psychische Störungen und Einschränkungen der gesundheitsbezogenen Lebensqualität nach sich zieht. Mit der vorliegenden Studie soll untersucht werden, in welchen konkreten Dimensionen die gesundheitsbezogene Lebensqualität bei Tinnituspatienten eingeschränkt ist, und wie sich diese Einschränkungen im Zeitverlauf ändern. Des Weiteren soll beurteilt werden, wie häufig Depressivität, Ängstlichkeit und Fatigue bei der Studienpopulation auftreten. Ein Vergleich mit Daten der Allgemeinbevölkerung bezüglich der gesundheitsbezogenen Lebensqualität, der Ausprägung von Depressivität, Ängstlichkeit und Fatigue wird durchgeführt.

120 Patienten mit Tinnitus und Indikation einer hyperbaren Sauerstofftherapie wurden zu drei Zeitpunkten untersucht: am Beginn (t1) und am Ende (t2) der zweiwöchigen Therapie sowie vier Wochen danach (t3). Eingesetzt wurden die Hospital Anxiety and Depression Scale (HADS), das Multidimensional Fatigue Inventory (MFI) und der Lebensqualitätsfragebogen European Organization for Research and Treatment Quality of Life Questionnaire (EORTC QLQ-C30).

Im Vergleich zu Personen der Allgemeinbevölkerung sind die Tinnituspatienten in allen Bereichen der gesundheitsbezogenen Lebensqualität beeinträchtigt, am stärksten in den Skalen Soziale, Kognitive, Emotionale und Rollen-Funktionsfähigkeit sowie im Bereich finanzieller Schwierigkeiten mit hohen Effektstärken um 1,5. Im Laufe der Therapie verbessern sich die Werte, so dass sich der Abstand zum Niveau der Allgemeinbevölkerung etwa halbiert.

Die differenzierte Einschätzung von Fatigue mit dem MFI zeigte in den Bereichen Mentale Fatigue, Allgemeine Fatigue und Körperliche Fatigue die massivsten Einschränkungen bei den Tinnituspatienten, wogegen die Dimensionen Reduzierte Aktivität und Reduzierte Motivation weniger betroffen waren.

Die zu t1 erfassten HADS-Werte für Angst und Depressivität liegen in der gleichen Größenordnung wie die HADS-Mittelwerte, welche in anderen Studien bei Tinnituspatienten ermittelt wurden.

Zwar gestatten die Ergebnisse wegen des Fehlens einer Kontrollgruppe keine Schlüsse über die Wirksamkeit der hyperbaren Sauerstofftherapie, sie zeigen aber das vielfältige Belastungsspektrum der Patienten (insbesondere im psychosozialen Bereich) auf und vermitteln Ansatzpunkte für unterstützende Angebote. 



\section{Einführung}

\section{Psychische Belastung und Lebensqualität bei Tinnituspatienten}

\section{Einleitung}

Geräusche sind wichtig um unsere Umwelt wahrzunehmen und zu analysieren. Im Innenohr, speziell in der Cochlea weisen die etwa 16.000 Haarzellen ständige mechanische und elektrische Aktivitäten als Reaktion auf Geräusche auf. Diese Aktivitäten werden als elektrische Impulse in den 30.000 Fasern des Hörnervs an die Hörrinde im Gehirn weitergegeben. Erst im Gehirn entstehen aus Geräuschen Sprache oder eine angenehme Melodie.

Tinnitus ist eine akustische Wahrnehmung des Menschen, die zusätzlich zum Schall, der auf das Ohr wirkt, wahrgenommen wird. Er ist ein Höreindruck (Ohrgeräusch, Ohrenklingen, Ohrensausen), der nicht auf der Stimulation durch einen äußeren Schallreiz beruht [1,2].

Diese Wahrnehmung beruht auf einer Störung der Hörfunktion des Menschen. Die Art der scheinbar wahrgenommenen Geräusche ist sehr vielfältig.

\section{Epidemiologie des Tinnitus}

Nach einer epidemiologischen Studie der deutschen Tinnitus-Liga e. V. sind knapp drei Millionen Deutsche von Tinnitus betroffen. Das entspricht vier Prozent der Gesamtbevölkerung. Auf Grund der Häufigkeit des Auftretens bezeichnet die Präsidentin der Deutschen Tinnitus-Liga den Tinnitus auch als Volkskrankheit.

1,5 Millionen Bundesbürger haben einen mittelschwer bis schwer ausgeprägten Tinnitus. Die Deutscher Tinnitus-Liga berichtet über eine jährliche Zuwachsrate für chronischen Tinnitus von 270.000 Betroffenen [3].

Laut Tönnies leiden sogar mehr als drei Millionen Menschen unter Ohrgeräuschen. Dabei ist bei mindestens einer halben Million Menschen der Tinnitus so unerträglich, dass sie kein normales Leben mehr führen können. Jährlich werden 340000 Bundesbürger neu von chronischem Tinnitus betroffen [4].

Bei 35-45 Prozent aller Erwachsenen über 17 Jahren tritt unter den Lebensbedingungen einer Industriegesellschaft zu irgendeinem Zeitpunkt ein transientes oder permanentes Ohrgeräusch unterschiedlicher Lautheit auf. Dieses Ohrgeräusch hält bei 15 Prozent länger als fünf Minuten an. Eine in Deutschland in der jüngeren 
Vergangenheit durchgeführte Erhebung analysierte die Verbreitung des Tinnitus. Eine telefonische Befragung von ca. 3000 Personen kam zu dem Ergebnis, dass rund ein Viertel der (über zehn Jahre alten) Befragten in der Vergangenheit schon einmal unter Ohrgeräuschen litt oder derzeit Ohrgeräusche hat. Vier Prozent der Befragten gaben zum Zeitpunkt der Erhebung an, aktuell unter Ohrgeräuschen zu leiden. Der überwiegende Teil hatte diese schon länger als drei Monate. Eine starke Beeinträchtigung durch die Ohrgeräusche gaben weniger als ein Drittel der Betroffenen an [5]. Im Jahr 2003 führte das Robert Koch Institut eine telefonische Befragung an 8300 Personen im Alter zwischen 18 und 79 Jahren zum Auftreten von beeinträchtigendem Tinnitus in den letzten sieben Tagen und zu dessen Dauer durch. Auch hier wiesen die Ergebnisse daraufhin, dass der überwiegende Teil der von Tinnitus Betroffenen diesen schon länger als drei Monate hatte. So gaben nur 6,5 Prozent der weiblich Befragten und 5,5 Prozent der männlichen einen bestehenden Tinnitus von weniger als drei Monaten an. Dagegen hatten 25,5 Prozent der befragten weiblichen Bevölkerung und 28,9 Prozent der männlichen einen Tinnitus von mehr als drei Monaten [6].

Etwa acht Prozent der unter Tinnitus leidenden Menschen fühlen sich durch das Ohrgeräusch in ihrem Alltag belästigt oder weisen Schlafstörungen auf [7]. Auch bei Kindern und Jugendlichen tritt zunehmend häufiger bereits ein Tinnitus auf.

Nicht selten ist ein Hörsturz mit der Ausbildung eines Tinnitus vergesellschaftet. In der Literatur wird die jährliche Inzidenz des Hörsturzes für Industrienationen mit 10 bis 20 pro 100000 Menschen der Bevölkerung angegeben. Das würde für Deutschland bedeuten, dass ca. 15000 Menschen pro Jahr an einem Hörsturz erkranken [6]. Da die Wahrscheinlichkeit des Tinnitus mit steigendem Alter zunimmt und die Anzahl der Älteren wächst, ist in Zukunft mit einer Zunahme der Betroffenen zu rechnen.

\section{Genese des Tinnitus}

Tinnitus kann im gesamten auditorischen System entstehen. Die Ursache des Tinnitus beruht in der Regel auf einem primären pathophysiologischen Prozess im Ohr (peripherer Tinnitus). Die pathophysiologischen Prozesse führen über hochempfindliche auditorische Rückkoppelungsmechanismen zum Symptom Tinnitus [8, 9 , 10]. Ursachen für eine Schädigung des Innenohres sind das Lärm- bzw. Knalltrauma, der Hörsturz sowie Störungen der Durchblutung (striae vascularis) und degenerative Veränderungen mit Schädigung der inneren und äußeren Haarzellen in 
der Cochlea. Aufgrund der vielfältigen Erscheinungsformen und der Komplexität der zugrunde liegenden Mechanismen existieren über die Entstehung des Tinnitus viele Hypothesen (Störung des lonentransports, unkontrollierte Transmitterfreisetzung an der Sinneszelle, Schädigung im Verlauf der Hörbahn oder im Hörzentrum und viele andere).

Wahrscheinlich handelt es sich beim Tinnitus um eine Art Grundaktivität unseres Hörsystems, die wegen ihres geringen Pegels und spezieller Unterdrückungsmechanismen (Filter) normalerweise nicht wahrgenommen wird. Durch eine Fehlinformation aus dem Ohr über die Hörbahn zum Gehirn und Beeinträchtigung des Filters wird die Grundaktivität als Geräusch wahrgenommen. Über interne Rückkopplung kann sich dieses Geräusch verselbstständigen und gelangt ins Bewusstsein, da das Gehirn nicht mehr fähig ist, es primär zu unterdrücken. Das Geräusch wird emotional als beunruhigend oder bedrohend empfunden und immer mehr in das Zentrum der Aufmerksamkeit des Betroffenen gerückt. Man spricht dann von einer sekundären Zentralisierung des Tinnitus [11].

Die zentralnervöse Verarbeitung führt häufig zu pathologisch übersteigerten ReizAntworten. Für diese pathologisch übersteigerten Reiz-Antworten werden heute besondere zentrale neurophysiologische Verarbeitungsmechanismen des Tinnitusreizes auch auf der kognitiven Ebene des Gehirns verantwortlich gemacht (kognitive Sensibilisierung) [12]. Dies bewirkt eine übersteigerte Aufmerksamkeitslenkung zum Tinnitus, Angstauslösung und Schlafstörungen.

Tinnitus ist für sich keine Krankheit sondern ein Symptom. Neben den o.g. Ursachen sind vielfältige andere möglich.

Zu den Ursachen gehören:

- Mittelohrerkrankungen mit Störung der Schallübertragung

- Sauerstoffmangelversorgung des Innenohres selber, aber auch infolge von Schlafapnoe oder Verspannung der Hals- und Nackenmuskulatur

- Virusinfektionen

- Funktionsstörungen der Halswirbelsäule

- Craniomandibuläre Dysfunktionen

- Nikotin- und Alkoholabusus

- Vergiftungen

- Medikamente ( z.B. zur Malariaprophylaxe, nichtsteroidale Antirheumatika) 
- Tumoren der Gehörnerven

- Autoimmun- und Stoffwechselerkrankungen

- Stress und psychosomatische Erkrankungen.

Dennoch tritt Tinnitus häufig ohne derzeit erkennbare medizinische Ursachen auf. Da viele Patienten in stressigen Lebensphasen und Situationen, in denen es innen psychisch schlecht geht, verstärkte Ohrgeräusche wahrnehmen, ist ein psychosomatischer Einfluss annehmbar. Die den Tinnitus beeinflussenden psychosozialen Faktoren sollen sensibilisierend auf der kognitiven Ebene wirken und auf diese Weise die Wahrnehmung eines sekundär zentralisierten Tinnitus steigern [13, 14, 15]. Eine primär zentrale Ursache des Tinnitus ist selten.

Bei erkennbaren Ursachen lassen sich diese beheben oder behandeln. Häufiger werden jedoch keine organischen Ursachen gefunden. In beiden Fällen kann der Tinnitus wieder völlig abklingen, er kann aber auch bleiben.

\section{Tinnitusformen}

Entsprechend der S1 Leitlinie Tinnitus von 2010 unterscheidet man beim Entstehungsmechanismus des Tinnitus zwischen objektivem und subjektivem Tinnitus, hinsichtlich des Zeitverlaufes zwischen akutem und chronischem Tinnitus sowie nach dem Ort der Entstehung: äußeres Ohr - Mittelohr - Innenohr - Hörnerv Gehirn einschließlich Hörbahn [16].

Objektiv ist der Tinnitus, der nicht nur vom Betroffenen selbst, sondern auch von Außenstehenden gehört wird, z. B. durch Gefäßerkrankungen im Ohr (vaskuläres Strömungsgeräusch) oder in unmittelbarer Ohrumgebung, z. B. Muskelkontraktionen, hervorgerufen wird. Diese Tinnitusform ist selten.

Die meisten von Tinnitus Betroffenen leiden an einem subjektiven Tinnitus, welcher nur vom Betroffenen selbst, aber nicht von Außenstehenden wahrgenommen wird. Es liegt eine fehlerhafte Informationsbildung im auditorischen System ohne Einwirkung eines akustischen Reizes vor. Ursachen dafür sind z. B. in der Cochlea, im Hirnstamm oder der zentralen Hörbahn zu suchen.

Die Pathophysiologie ist teilweise noch ungeklärt. Wahrscheinliche Ursachen sind Defekte im Haarzellbereich, Störungen der neuralen Spontanaktivität der Hörnerven- 
fasern sowie krankhafte Veränderungen der biochemischen Vorgänge in den Haarzellen oder Unterbrechung des Kontaktes der Haarzellen zur Tectorialmembran.

Akut nennt man gemäß Leitlinie den Tinnitus, der bis zu drei Monate besteht. Chronisch ist jeder Tinnitus mit einer Dauer von mehr als drei Monaten.

Die Dauer der Symptomatik erlaubt keinen Rückschluss auf die individuelle Ätiologie oder die subjektive Belastung durch den Tinnitus.

\section{Schweregrade des Tinnitus}

Wichtig ist insbesondere bei der Therapie eine Einteilung nach Schweregrad.

Nach der Leitlinie "Tinnitus“ der Deutschen Gesellschaft für HNO-Heilkunde, Kopfund Halschirurgie wird der Schweregrad in mehrere Kategorien eingeteilt, wobei die Einteilung des Schweregrades nach Biesinger et al. 1998 [17] eine klinischpraktische ist, sich an der klinischen Situation orientiert und die Auswirkung des Ohrgeräusches bei den Betroffenen im beruflichen und privaten Bereich berücksichtigt:

Grad 1: $\quad$ Der Tinnitus ist gut kompensiert, kein Leidensdruck.

Grad 2: Der Tinnitus tritt hauptsächlich in Stille in Erscheinung und wirkt störend bei Stress und Belastungen.

Grad 3: Der Tinnitus führt zu einer dauernden Beeinträchtigung im privaten und beruflichen Bereich. Es treten Störungen im emotionalen, kognitiven und körperlichen Bereich auf.

Grad 4: Der Tinnitus führt zur völligen Dekompensation im privaten Bereich, Berufsunfähigkeit.

Die Gradeinteilung nach Goebel und Hiller beruht auf einer strukturierter Anamnese (z. B. strukturiertes Tinnitus-Interview (STI)) [18].

Grad 1: $\quad$ Kein Leidensdruck.

Grad 2: Der Tinnitus tritt hauptsächlich in der Stille auf, ist störend bei Stress und psychischen Belastungen.

Grad 3: Der Tinnitus führt zu dauernder Beeinträchtigung im kognitiven, emotionalen körperlichen Bereich.

Grad 4: Der Tinnitus führt zur massiven psychischen Dekompensation. 
Man unterscheidet zwischen kompensiertem und dekompensiertem Tinnitus.

Bei Tinnitus Grad 1 und 2 spricht man vom kompensierten Tinnitus, Grad 3 und 4 entsprechen einem dekompensierten Tinnitus.

Kompensiert ist der Tinnitus, wenn er keine relevanten Folgesymptome im körperlichen, kognitiven oder emotionalen Bereich beim Patienten hervorruft. Es besteht kein oder nur geringer Leidensdruck. Wenn derartige Folgen eingetreten sind, spricht man von einem dekompensierten Tinnitus. Es besteht ein hoher Leidensdruck und die Lebensqualität ist wesentlich beeinträchtigt.

Eine Erfassung des Schweregrades des Tinnitus sowie möglicher Sekundärsymptome kann durch ein standardisiertes Kurzinterview geschehen [19]. Die quantitative Erfassung der Lautheit und des Belästigungsgrades kann durch visuelle Analogskalen erfolgen [20]. Diese können auch zur Verlaufs- und Therapiekontrolle eingesetzt werden. Eine psychologische Diagnostik ist zur Erfassung von Begleitstörungen, insbesondere bei dekompensiertem, chronischem Tinnitus zu empfehlen.

\section{Psychische Belastungen bei Tinnitus}

Ohrgeräusche sind in den meisten Fällen weder von ihrer Ursache noch von ihren Auswirkungen her lebensbedrohend.

Medizinisch gesehen gilt der Tinnitus daher weniger als Krankheit, sondern als Krankheitssymptom. Dieses kann jedoch unbehandelt im Laufe der Jahre zu schwerwiegenden gesundheitlichen Problemen führen.

In etwa sieben Prozent der Fälle kommt es nach dem ersten Auftreten zu einer weiteren Verschlechterung der Ohrgeräusche. Tinnitus führt aber durch den Verlust der Stille, den ständigen Geräuschen und dem Gefühl, ihnen nicht entrinnen zu können, bei nicht wenigen Betroffenen zu körperlichen und psychischen Auswirkungen, die Leben und Arbeit beeinträchtigen. Damit führen sie zum dekompensierten Tinnitus.

Patienten mit einem dekompensierten Tinnitus sind sozial beeinträchtigter, depressiver bis hin zur Suizidalität und besitzen eine ineffektivere Krankheitsverarbeitung [21]. Im Vergleich zu Patienten mit einem kompensierten Tinnitus liegt bei Patienten mit dekompensierten Tinnitus eine deutlich höhere somatische Multimorbidität, insbesondere in der Vergesellschaftung mit Hyperakusis und HerzKreislauf-Erkrankungen vor [21]. 
Bei 0,5 Prozent der Erwachsenen hat der Tinnitus den Stellenwert einer eigenständigen Erkrankung, die zu einer wesentlichen Beeinträchtigung der gesundheitsbezogenen Lebensqualität durch Konzentrations- und Schlafstörungen, reaktive Depressionen und Angstzustände führt. Häufig führt ein dekompensierter Tinnitus zu länger anhaltender Arbeitsunfähigkeit, welche mit finanziellen Einbußen verbunden ist und somit die soziale Situation des Betroffenen verschlechtern kann. Bezogen auf den Bevölkerungsanteil der Erwachsenen in Deutschland umfasst diese Gruppe ca. 300.000 Personen. Die Mehrzahl der von Tinnitus betroffenen Patienten können jedoch auf Dauer die Ohrgeräusche gut kompensieren und leiden unter keiner bzw. lediglich einer geringen Einschränkung der Lebenssituation [7].

Zwischen subjektiver Belastung durch Ohrgeräusche und psychischen Störungen wurde ein stark ausgeprägter Zusammenhang nachgewiesen [22, 23]. Menschen mit dekompensiertem Tinnitus haben im Vergleich zur Allgemeinbevölkerung eine deutlich erhöhte psychische Komorbidität [24]. Bei Tinnituspatienten steht der Tinnitusschweregrad in Beziehung zum Schweregrad von Angststörungen und Depressionen [25]. So weisen Patienten mit dekompensiertem Tinnitus ein fünf- bis siebenfaches Risiko bezüglich Depressivität und Ängstlichkeit auf gegenüber Patienten mit kompensierten Tinnitus [26, 27 28, 29]. Zur Prävalenz psychischer Störungen bei Tinnituspatienten wurden folgende Prozentzahlen ermittelt: 18-39 Prozent depressive Störungen, 11-19 Prozent Angststörungen, 8-34 Prozent somatoforme Störungen und 19 Prozent Persönlichkeitsstörungen [25, 30, 31]. Personen mit dekompensiertem Tinnitus sind in der Regel stark in ihrer Lebensqualität und der Teilhabe am Alltagsgeschehen beeinträchtigt [26].

Tinnitusassoziierte Depressivität hat ebenso eine sozialmedizinische Relevanz. Patienten mit dekompensiertem Tinnitus haben eine geringere Wahrscheinlichkeit, ihre Berufstätigkeit wieder aufzunehmen [32]. Die Lebensqualität ist durch Konzentrations- und Schlafstörungen, reaktive Depression und Angstzustände eingeschränkt [33]. Tinnituspatienten, die gleichzeitig unter Angst und Depressivität leiden, sind in ihrer Lebensqualität stärker beeinträchtigt, als solche, die nur unter Angst oder Depressivität leiden [34]. Die Lebensqualität ist besonders bei denjenigen Tinnituspatienten beeinträchtigt, die über reduzierte Konzentration, Depression und emotionale Probleme in der Beziehung mit der Familie, Freunden und Kollegen klagen [35]. 
Neben den Studien zur psychischen Belastung existieren auch Untersuchungen zur gesundheitsbezogenen Lebensqualität bei Tinnituspatienten. Diese nutzen tinnitusspezifische Fragebögen (vgl. zusammenfassend [36]) oder generische, krankheitsübergreifende Instrumente. Unter Nutzung des Fragebogens Short Form 36 (SF-36) haben Studien in Großbritannien und in den USA nachgewiesen, dass die gesundheitsbezogene Lebensqualität von Tinnituspatienten in allen Dimensionen des SF-36 eingeschränkt ist [37, 38, 39]. Dabei nahmen die Einbußen in der Lebensqualität mit steigender Tinnitusschwere erwartungsgemäß zu, insbesondere in den Dimensionen Rollen-Funktionsfähigkeit und Schmerzen.

Es gibt eine Reihe von Studien, die eine hohe psychische Belastung bei Tinnituspatienten belegen, besonders Angst und Depressivität.

Es gibt wenige Studien, die die Problematik Fatigue und Tinnitus untersuchen. Auch eine differenzierte Lebensqualität-Analyse mit einzelnen Symptomen, wie es mit dem European Organization for Research and Treatment Quality of Life Questionnaire (EORTC) möglich ist, fehlt weitgehend. Studien mit dem SF-36 liefern zwar Aussagen zu verschiedenen Bereichen der Lebensqualität inklusive Vitalität, eine differenzierte Auffächerung des Bereichs Fatigue, wie sie mit einem multidimensionalen Fatigue-Bogen möglich ist, fehlt. Schließlich handelt es sich bei den in der Literatur beschriebenen Studien um Querschnitts-Studien. Die Veränderungen der psychischen Belastung und der Lebensqualität im Zeitverlauf und während einer bzw. infolge einer Therapie sind bisher kaum untersucht worden.

In einzelnen Studien wird versucht, die Wirksamkeit von Therapien nachzuweisen, dann aber i. A. ohne Bezug zu den Werten der Allgemeinbevölkerung. Aus diesen Gründen soll mit der vorliegenden Studie die gesundheitsbezogene Lebensqualität, Fatigue und das psychische Befinden im Verlauf untersucht und ein Vergleich mit Daten der Allgemeinbevölkerung durchgeführt werden. 


\section{Literaturverzeichnis}

1 Hesse G. Tinnitus. Stuttgart: Thieme, 2008

2 Kempf HG, Zenner HP. Tinnitus. In: Zenner H P, Hrsg. Praktische Therapie von HNO-Krankheiten. Stuttgart: Schattauer, 2008; 133-141

3 Deutsche Tinnitus-Liga e.V. Epidemiologische Studie der Deutschen Tinnitus-Liga: http://www.tinnitus-liga.de/Presse_News/presse3.pdf

4 Tönnies S. Leben mit Ohrgeräuschen. Selbsthilfe bei Tinnitus. Heidelberg: Asanger, 2001

5 Siedentop H, Kirchhoff D, Rychlik R. Tinnitus in der Bundesrepublik Deutschland. Abschlussbericht 1999. Institut für Empirische Gesundheitsökonomie. Burscheid, 1999

6 Streppel M, Walger M, von Wedel H. Hörstörungen und Tinnitus.

Gesundheitsberichterstattung des Bundes, Heft 29. Berlin: Robert Koch Institut, 2006

7 Lenarz T. Epidemiologie. In: Feldmann H. Tinnitus: Faktor Industriegesellschaft. Stuttgart: Thieme, 1998; 77-83

8 Hesse G. Neurootologisch-psychosomatische Habituationstherapie. Therapieansätze bei chronischem Tinnitus. HNO 2008; 56: 686-693

9 Hesse $\mathrm{G}$, Andres R, Schaaf $\mathrm{H}$, Laubert A. DPOAE und laterale Inhibition bei chronischemTinnitus. HNO 2008; 56: 694-700

10 Preyer S, Bootz F. Tinnitusmodelle zur Verwendung bei der Tinnituscounsellingtherapie des chronischen Tinnitus. HNO 2005; 43: 338-351

11 Zenner HP. A systematic classification of tinnitus generator mechanisms. Int Tinnitus J 1998; 4: 109-113

12 Zenner HP, Pfister M, Birbaumer N. Tinnitus sensitization: Sensory and psychophysiological aspects of a new pathway of acquired centralization of chronic tinnitus. Otol Neurotol 2006; 27: 1054-1063

13 Fichter M, Goebel G. Psychosomatische Aspekte des chronischen komplexen Tinnitus. Deutsches Ärzteblatt 2006; 26: A1771-A1776

14 Georgiewa P, Klapp BF, Fischer F, Reisshauer A, Juckel G, Frommer J, Mazurek B. An integrative model of developing tinnitus based on recent neurobiological findings. Med Hypotheses 2006; 66: 592-600

15 Goebel G. Ohrgeräusche. Psychosomatische Aspekte des komplexen chronischen Tinnitus: Vorkommen, Auswirkungen, Diagnostik und Therapie. Quintessenz, München, 1992 
16 AWMF-Leitlinie Tinnitus. http://www.awmf.org/uploads/tx_szleitlinien/017064I_S1_Tinnitus.pdf

17 Biesinger E, Heiden C, Greimel V, Lendle T, Hoing R, Albegger K. Strategien in der ambulanten Behandlung des Tinnitus. HNO 1998; 46: 157-169

18 Goebel G, Hiller W. Verhaltensmedizinische Tinnitus-Diagnostik. Eine praktische Anleitung zur Erfassung medizinischer und psychologischer Merkmale mittels des strukturierten Tinnitus-Interviews (STI). Göttingen: Hogrefe, 2001

19 Goebel G. Verhaltensmedizinische Diagnostik des Tinnitus. Standardisiertes Tinnitus-Interview (STI). HNO aktuell 1994; 2: 281-288

20 Wilhelm T, Ruth S, Bock K, Lenarz T. Standardisierung und Qualitätssicherung am Beispiel Tinnitus. Laryngo-Rhino-Otol 1995; 74: 300-306

21 Stobik C, Weber RK, Münt TF, Frommer J. Psychosomatische Belastungsfaktoren bei kompensiertem und dekompensiertem Tinnitus. Psychother Psychosom Med Psychol. 2003; 8: 344-352

22 Zirke N, Goebel G, Mazurek B. Tinnitus and psychological comorbidities. HNO. 2010 Jul; 58: 726-732

23 Schaaf H, Dölberg D, Seling B, Märtner M. Komorbidität von Tinnituserkrankungen und psychiatrischen Störungen. Nervenarzt. 2003 Jan; 74: 72-75

24 Jacobi F, Wittchen HU, Holting C, Höfler M, Pfister H, Müller N, Lieb R. Prevalence, co-morbidity and correlates of mental disorders in the general population: results from the German Health Interview and Examination Survey (GHS). Psychol Med 2004; 34: 597-611

25 Zoger S, Svedlund J, Holgers KM. Relationship between tinnitus severity and psychiatric disorders. Psychosom 2006; 47: 282-288

26 Härter M, Maurischat C, Weske G, Laszig R, Berger M. Psychische Belastungen und Einschränkungen der Lebensqualität bei Patienten mit Tinnitus. HNO 2004; 52:125-131

27 D'Amelio R, Archonti C, Scholz S, Falkai P, Plinkert PK, Delb W. Akuter Tinnitus. Psychische Korrelate und Ausmaß der Belastung bei Patienten. HNO 2004; 52 : 599-603

28 Svitak M, Rief W, Goebel G. Kognitive Therapie des chronischen dekompensierten Tinnitus. Psychother 2001; 46: 317-325

29 Schaaf H, Eipp C, Deubner R, Hesse G, Vasa R, Gieler U. Psychosoziale Aspekte der Krankheitsverarbeitung bei Tinnitus- und Psoriasis-Patienten. Eine Vergleichsstudie hinsichtlich Suizidalität, Ängstlichkeit und Depressivität. HNO. 2009 Jan;57: 57-63 
30 Belli S, Belli H, Bahcebasi T, Ozcetin A, Alpay E, Ertem U. Assessment of psychopathological aspects and psychiatric comorbidities in patients affected by tinnitus. Eur Arch Oto-Rhino-Laryngol 2008; 265: 279-285

31 Konzag TA, Rubler D, Bandemer-Greulich U, Frommer J, Fikentscher E. Tinnitusbelastung und psychische Komorbidität bei ambulanten subakuten und chronischen Tinnituspatienten. Z Psychosom Med Psychother 2005; 51: 247-260

32 Holgers K-M, Erlandsson SI, Barrenäs M-L. Predictive factors for the severity of tinnitus. Audiol 2000; 39: 284-291

33 Lenarz T. Epidemiologie. In: H. Feldmann. Tinnitus: Grundlagen einer rationalen Diagnostik und Therapie. Stuttgart: Thieme; 1998; 77-83

34 Bartels $\mathrm{H}$, Middel BL, van der Laan BFAM, Staal MJ, Albers FWJ. The additive effect of co-occurring anxiety and depression on health status, quality of life and coping strategies in help-seeking tinnitus sufferers. Ear \& Hearing 2008; 29: 947956

35 Erlandsson SI, Hallberg IR-M. Prediction of quality of life in patients with tinnitus. Brit J Audiol 2000; 34: 11-20

36 Kamalski DM, Hoekstra CE, van Zanten BG, Grolmann W, Rovers MM. Measuring disease-specific health-related quality of life to evaluate treatment outcomes in tinnitus patients: a systematic review. Otolaryngol -Head Neck Surgery 2010; 143: 181-185

37 Nondahl DM, Cruickshanks KJ, Dalton DS, Klein R. The impact of tinnitus on quality of life in older adults. J Am Acad Audiol 2007; 18: 257-266

38 Davis AC, Roberts H. Tinnitus and health status: SF-36 profile and accident prevalence. In: Reich GE, Vernon JA (Eds). Proceedings of the fifth international tinnitus seminar, 1995. Portland, OR: American Tinnitus Association, 257-265

39 Wilson C, Lewis P, Stephens D. The Short Form 36 (SF36) in a specialist tinnitus clinic. Int J Audiol 2002; 41: 216-220 

3. Publikation 


\title{
Psychische Belastung und Lebensqualität bei Tinnituspatienten
}

\author{
Mental Distress and Quality of Life in Tinnitus Patients
}

\author{
Autoren \\ S. Hesse ${ }^{1}$, A. Meyer ${ }^{2}$, S. Singer ${ }^{3}$, A. Hinz ${ }^{2}$ \\ Institute \\ ${ }^{1}$ Helios-Klinikum Borna, Klinik für Innere Medizin, Borna
${ }^{2}$ Abteilung für Medizinische Psychologie und Medizinische Soziologie, Universität Leipzig
${ }^{3}$ Institut für Medizinische Biometrie, Epidemiologie und Informatik, Universität Mainz
}

Schlüsselwörter

psychische Belastung

- Komorbidität

- Lebensqualität

- Tinnitus

Distress

Key words

mental load

comorbidity

quality of life

tinnitus

mental distress

eingereicht 16. Februar 2012

akzeptiert 12. August 2012

Bibliografie

DOI http://dx.doi.org/

10.1055/s-0032-1323731

Online-Publikation: 22.10.2012

Laryngo-Rhino-Otol 2012; 91:

774-781 @ Georg Thieme

Verlag KG Stuttgart · New York

ISSN 0935-8943

Korrespondenzadresse

Prof. Andreas Hinz

Abteilung für Medizinische

Psychologie und Medizinische

Soziologie

Universität Leipzig

Philipp-Rosenthal-Straße 55

04103 Leipzig

andreas.hinz@medizin.uni-

leipzig.de

\section{Zusammenfassung}

\section{$\nabla$}

Hintergrund: Tinnitus ist eine Erkrankung mit hoher Prävalenz, welche häufig psychische Störungen nach sich zieht. Mit der vorliegenden Studie sollte untersucht werden, in welchen konkreten Dimensionen die gesundheitsbezogene Lebensqualität bei Tinnituspatienten eingeschränkt ist, und wie sich diese Einschränkungen während und nach Therapie, einschließlich einer hyperbaren Sauerstofftherapie, ändern.

Material und Methoden: 120 ambulant oder stationär behandelte Patienten mit Tinnitus wurden zu 3 Zeitpunkten untersucht: am Beginn (t1) und am Ende ( $\mathrm{t} 2$ ) der 2-wöchigen Therapie sowie 4 Wochen danach ( $t 3$ ). Eingesetzt wurden die Hospital Anxiety and Depression Scale, das Multidimensional Fatigue Inventory und der Lebensqualitätsfragebogen EORTC QLQ-C30.

Ergebnisse: Im Vergleich zu Personen der Allgemeinbevölkerung waren die Tinnituspatienten

\section{Einleitung}

Tinnitus bezeichnet alle Formen von akustischen Wahrnehmungen, die nicht durch äußere Schallquellen bedingt sind („Ohrgeräusche“) [1,2]. Diese beruhen auf einer Störung der Hörfunktion. Tinnitus kann grundsätzlich bei fast jeder Ohrerkrankung begleitend auftreten. In diesen Fällen spricht man von einem symptomatischen Tinnitus im Unterschied zu dem ohne erkennbare Ursache akut auftretenden idiopathischen Tinnitus. Jährlich erleben in Deutschland rund 10 Millionen Menschen in irgendeiner Form einen akuten Tinnitus [3]; die Häufigkeit von chronischem Tinnitus wird auf rund 3 Millionen geschätzt, entsprechend rund $4 \%$ der Bevölkerung [4]. Dabei ist bei mindestens einer halben Million Menschen der Tinnitus subjektiv so unerträglich, dass sie kein normales Leben mehr führen können. Tinni- in allen Bereichen beeinträchtigt, am stärksten in den Skalen Soziale, Kognitive, Emotionale und Rollen-Funktionsfähigkeit sowie im Bereich finanzieller Schwierigkeiten mit hohen Effektstärken um 1,5. Im Laufe der Therapie verbesserten sich die Werte, sodass sich der Abstand zum Niveau der Allgemeinbevölkerung etwa halbierte. Die durch die Patienten direkt eingeschätzte Verbesserung durch die Therapie korrespondierte nur geringfügig mit der Verbesserung, die sich aus dem Vergleich der Prä- und Post-Fragebögen ergab.

Schlussfolgerung: Zwar gestatten die Ergebnisse wegen des Fehlens einer Kontrollgruppe keine Schlüsse über die Wirksamkeit der hyperbaren Sauerstofftherapie, sie zeigen aber das vielfältige Belastungsspektrum der Patienten (insbesondere im psychosozialen Bereich) auf und vermitteln Ansatzpunkte für unterstützende Angebote.

tus kann mit einem Hörsturz vergesellschaftet sein, welcher in Deutschland jährlich rund 15000 Personen betrifft [5]. Eine Studie aus den USA bei älteren Personen (mittleres Alter: 69 Jahre) kam auf eine Häufigkeit von 25\% der Personen mit zumindest leichtem Tinnitus [6]. Ein neuerer Review-Artikel [7] kommt jedoch zu dem Schluss, dass die Lebenszeitprävalenz von Tinnitus nur rund $10 \%$ beträgt.

„Auch wenn Tinnitus von medizinischer Seite nicht als eigenes Krankheitsbild gesehen wird, können Betroffene doch in erheblichem Ausmaß unter dem oft als harmlos betrachteten Symptom leiden." [8]. Tinnitus führt durch den Verlust der Stille, durch die ständigen Geräusche und das Gefühl, ihnen nicht entrinnen zu können, bei vielen Betroffenen zu körperlichen und psychischen Problemen, die Leben und Arbeit massiv beeinträchtigen. Man spricht dann von dekompensiertem Tinnitus [9]. 
Zwischen subjektiver Belastung durch Ohrgeräusche und psychischen Störungen wurden enge Zusammenhänge nachgewiesen [10-12]. Insbesondere Patienten mit dekompensiertem Tinnitus haben im Vergleich zur Allgemeinbevölkerung eine deutlich erhöhte psychische Komorbidität [13] und weniger effektive Krankheitsbewältigungsstrategien [14]. Der Tinnitusschweregrad steht in Beziehung zu psychischen Störungen und zum Schweregrad von Angststörungen und Depressionen [15]. Zur Prävalenz psychischer Störungen bei Tinnituspatienten wurden folgende Prozentzahlen ermittelt: 18-39\% depressive Störungen, 11-19\% Angststörungen, 8-34\% somatoforme Störungen und 19\% Persönlichkeitsstörungen [14-17]. Insbesondere bei chronisch dekompensiertem Tinnitus kann eine kognitiv-behaviorale Therapie angezeigt sein [18].

Neben den Studien zur psychischen Belastung existieren auch Untersuchungen zur gesundheitsbezogenen Lebensqualität bei Tinnituspatienten. Diese nutzen tinnitusspezifische Fragebögen (vgl. zusammenfassend [19]) oder generische, krankheitsübergreifende Instrumente. Unter Nutzung des Fragebogens SF-36 haben Studien in Großbritannien und in den USA nachgewiesen, dass die Lebensqualität von Tinnituspatienten in allen Dimensionen des SF-36 eingeschränkt ist $[6,20,21]$. Dabei nahmen die Einbußen in der LQ mit steigender Tinnitus-Schwere erwartungsgemäß zu, insbesondere in den Dimensionen Rollen-Funktionsfähigkeit und Schmerzen. Die Lebensqualität von TinnitusPatienten ist durch Konzentrations- und Schlafstörungen, reaktive Depression und Angstzustände eingeschränkt [22], außerdem zeigen sich Probleme in der Beziehung mit Familie, Freunden und Kollegen [23].

Eine bei Tinnitus gelegentlich eingesetzte Therapieform war die hyperbare Sauerstofftherapie $(H B O)$, auch wenn diese Therapie in der aktuellen Tinnitus-Leitlinie [24] keine Berücksichtigung mehr findet. Das Einatmen von 100\% Sauerstoff bei erhöhtem Luftdruck bewirkt dabei eine Erhöhung des Sauerstoffpartialdrucks. Typisch sind Treatment-Protokolle mit $60 \mathrm{~min}$ HBO bei 2,0-2,5 bar. Nachgewiesen ist die positive HBO-Wirkung bei akutem Hörverlust [25], während es widersprüchliche Berichte über die kurz- und langfristige Wirksamkeit bei Tinnitus gibt [26-28]. Bei einer HBO-Studie mit 360 Tinnituspatienten [29] gaben $49 \%$ der Patienten eine Verbesserung an.

Über die Verläufe von psychischer Belastung und Lebensqualität bei Tinnituspatienten ist wenig bekannt. Generell handelt es sich entweder um Querschnittsstudien, welche eine Momentaufnahme der Lebensqualität der Patienten zeigen, typischerweise in einem Setting als Klinikpatienten, oder es wird andererseits versucht, die Wirksamkeit von Therapien nachzuweisen, dann aber i.a. ohne Bezug zu den Werten der Allgemeinbevölkerung. Außerdem liefern die Studien mit dem SF-36 zwar Aussagen zu verschiedenen Bereichen der Lebensqualität inklusive Vitalität, eine differenzierte Auffächerung des Bereichs Fatigue, wie sie mit einem multidimensionalen Fatigue-Bogen möglich ist, fehlt aber ebenso wie die Analyse der Häufigkeit gewisser Symptome, die durch den SF-36 nicht erfasst werden wie Appetitlosigkeit, Übelkeit/Erbrechen und Schlafprobleme. Aus diesen Gründen soll mit der vorliegenden Studie die gesundheitsbezogene Lebensqualität in einer Weise untersucht werden, die sowohl Verläufe sichtbar macht als auch Vergleiche mit der Allgemeinbevölkerung in einer differenzierten Weise gestattet. Die konkreten Zielstellungen der vorliegenden Arbeit sind die folgenden:

- Analyse von Lebensqualität, Fatigue und psychischer Belastung bei Tinnituspatienten durch Vergleiche mit der Allgemeinbevölkerung,
- Analyse von Verläufen der Lebensqualität während einer hyperbaren Sauerstofftherapie über 3 Messzeitpunkte sowie

- Prüfung der Wirkung soziodemografischer Variablen und Reha-Teilnahme auf die Lebensqualität.

\section{Material und Methoden}

\section{$\nabla$}

Ablauf der Studie

Im Zeitraum von 2004-2006 wurden 120 Patienten, die im Fachklinikum Brandis an einer hyperbaren Sauerstofftherapie (HBO) wegen Tinnitus teilnahmen, hinsichtlich Angst und Depressivität, Fatigue sowie gesundheitsbezogener Lebensqualität untersucht. Eingeschlossen wurden 97 Patienten, die im Rahmen einer Rehabilitationsmaßnahme mit komplexem Behandlungsprogramm eine HBO erhielten und 23 Patienten, die ambulant eine HBO ohne begleitende andere Therapiemaßnahmen durchführten. Alle Patienten hatten vor der Beantragung der HBO-Behandlung leitliniengerecht bereits eine rheologische Behandlung oder Therapie mit Glukokortikoiden ohne den gewünschten Therapieerfolg erhalten. Die Überprüfung der Indikationsstellung für die HBO-Therapie und der Ausschluss von Kontraindikationen erfolgte in dem Fachklinikum Brandis durch einen ausgebildeten Arzt für Hyperbarmedizin (GTÜM). Zum Zeitpunkt der Studie erfolgte die Stadieneinteilung der Tinnituspatienten entsprechend der Leitlinie von 1998. Dementsprechend wurden Patienten mit akutem und subakutem Tinnitus in die Studie aufgenommen. Patienten mit chronischem Tinnitus (nach damaliger Einteilung ab 12 Monate Symptomdauer) wurden nicht mittels HBO behandelt. Die HBO als Therapieoption galt für Patienten mit akutem Tinnitus mit und ohne Hörsturz. Da die Patienten zur HBO-Therapie von den Krankenkassen oder vom Rentenversicherungsträger oft erst im Stadium des subakuten Tinnitus zugewiesen wurden, sind auch diese Patienten mittels HBO behandelt worden. Der Entscheid über die Art der Behandlung (stationär in Form einer Rehabilitationsmaßnahme oder als ambulante HBO-Therapie) oblag dem Kostenträger. Die stationär behandelten Patienten erhielten neben der HBO-Behandlung Entspannungstherapie in Form von Progressiver Muskelrelaxation (PMR), Krankengymnastische Übungsbehandlung für die Halswirbelsäule in Kombination mit physikalischen Therapien, z.B. Packungen und Massagen, und sie nahmen an psychologisch geleiteten Seminaren zur Krankheitsbewältigung und allgemeinen Konditionierungstherapien teil.

Die Patienten wurden vor Durchführung der ersten HBO-Sitzung über die Studie informiert. Nach Einverständniserklärung wurde den Patienten der erste Fragebogen ausgehändigt und noch am gleichen Tag ausgefüllt zurückgegeben ( $\mathrm{t} 1$ ). Vor Beginn der HBO-Therapie erfolgte eine Untersuchung zur Beurteilung der Druckkammertauglichkeit. Hierdurch sollte das Risikopotenzial für die Patienten hinsichtlich Nutzen-/Risikobewertung so gering wie möglich gehalten werden. Bei der Therapie atmen die Patienten bei einem Druck von $240 \mathrm{kPa}$ reinen Sauerstoff über insgesamt $60 \mathrm{~min}$ in einer Druckkammer. Das Standarduntersuchungsprogramm umfasste eine ausführliche Anamnese unter besonderer Berücksichtigung der geplanten Sauerstoffexposition in hyperbarer Umgebung, eine umfassende körperliche Untersuchung unter besonderer Berücksichtigung der gasgefüllten Hohlräume und Hohlorgane auf die Druckwechsel-Belastung, ein Ruhe-EKG, eine Röntgenaufnahme des Thorax, eine Lungenfunktionsprüfung, eine Otoskopie und eine Prüfung der Tubendurchgängigkeit. 
Tab. 1 Soziodemografische Daten.

\begin{tabular}{|c|c|c|}
\hline & n & $\%$ \\
\hline \multicolumn{3}{|l|}{ Geschlecht } \\
\hline Männer & 76 & 63 \\
\hline Frauen & 44 & 37 \\
\hline \multicolumn{3}{|l|}{ Familienstand } \\
\hline ledig, allein lebend & 19 & 15,8 \\
\hline ledig, feste Partnerschaft & 4 & 3,3 \\
\hline verheiratet & 84 & 70,0 \\
\hline verwitwet & 3 & 2,5 \\
\hline geschieden & 10 & 8,3 \\
\hline \multicolumn{3}{|l|}{ höchster Schulabschluss } \\
\hline Hauptschule & 37 & 30,8 \\
\hline Realschule & 64 & 53,3 \\
\hline Fachschule & 5 & 4,2 \\
\hline Hochschule & 9 & 7,5 \\
\hline keine Angaben & 5 & 4,0 \\
\hline \multicolumn{3}{|c|}{ beruflicher Ausbildungsabschluss } \\
\hline ja & 101 & 84,2 \\
\hline nein & 16 & 13,3 \\
\hline keine Angaben & 3 & 2,5 \\
\hline \multicolumn{3}{|l|}{ Berufstätigkeit } \\
\hline erwerbstätig & 79 & 65,8 \\
\hline nicht erwerbtätig & 41 & 34,2 \\
\hline
\end{tabular}

Über einen Zeitraum von 2 Wochen erhielten die Patienten 10 HBO-Anwendungen. Unmittelbar nach Beendigung der HBOTherapie wurde den Patienten der Fragebogen erneut zur Beantwortung vorgelegt (t2). Ein dritter Fragebogen wurde den Patienten mit nach Hause gegeben, er sollte nach Ablauf von 4 Wochen ausgefüllt an das Klinikum zurückgesandt werden ( $t 3$ ). Alle 120 Patienten nahmen auch an der zweiten Fragebogenerhebung (t2) teil. An der dritten Fragebogenerhebung (t3) beteiligten sich 98 der 120 Patienten $(81,7 \%)$.

\section{Fragebögen}

Angst und Depressivität. Zur Erfassung von Angst und Depressivität wurde die deutsche Version der Hospital Anxiety and Depression Scale (HADS) eingesetzt [30]. Die HADS enthält 14 Items mit 4-stufigen Antwortmöglichkeiten. Je 7 Items fließen in die Angst- bzw. Depressivitäts-Skala ein, der jeweilige Gesamtwert wird durch Addition der Einzelscores gebildet (Wertebereich $0-21$ ). Dabei werden Summenwerte $\leq 7$ als unauffällig, Werte von 8 bis 10 als grenzwertig und Werte ab 11 Punkte als auffällig bezeichnet. Die HADS ist psychometrisch vielfach erprobt [31]

Fatigue. Zur differenzierten Erfassung von Fatigue wurde das Multidimensional Fatigue Inventory (MFI-20) verwendet [32]. Der Fragebogen umfasst 5 Dimensionen: Allgemeine Fatigue, Körperliche Fatigue, Reduzierte Aktivität, Reduzierte Motivation und Mentale Fatigue. Jede der 5 Dimensionen wird aus 4 Items (mit 5-stufigem Antwortformat) gebildet, was auf Wertebereiche von 4 bis 20 führt. Es lässt sich ein Gesamtwert mit dem Wertebereich $20-100$ bilden.

Gesundheitsbezogene Lebensqualität. Die Lebensqualität wurde mit dem Fragebogen EORTC QLQ-C30 [33] erhoben. Dieser Fragebogen wurde zur Erfassung der Lebensqualität bei Krebspatienten entwickelt. Gegenüber anderen Lebensqualitätsfragebögen wie dem SF-36 hat er den Vorzug, dass er neben den Funktionsskalen verschiedene körperliche Symptome (vgl. $\odot$ Tab. 2) differenziert erfasst. Der Fragebogen enthält 30
Items, welche zu Funktions-, Symptom- und Globalskalen zusammengefasst werden. Die Items (mit 4-stufigem bzw. 7-stufigem Antwortformat) umfassen den körperlichen und psychischen Funktionsstatus, Beeinträchtigungen im Alltagsleben sowie Symptome wie Übelkeit, Schlafstörungen, Verdauungsprobleme und Erschöpfung. Außerdem werden Gesundheitszustand und Lebensqualität eingeschätzt. Die Skalen sind sämtlich auf den Bereich von 0 bis 100 normiert. Hohe Werte in den Funktionsskalen und in der Skala der globalen Lebensqualität stehen für eine hohe Lebensqualität; hohe Werte in den Symptomskalen bedeuten dagegen ausgeprägte Symptombelastungen. Für die integrierende Darstellung wurde ein (die Polungen berücksichtigender) Gesamtwert berechnet, der ebenfalls auf den Bereich 0-100 normiert ist [34].

Weitere Daten. Soziodemografische Variablen (Alter, Geschlecht, Familienstand, Schulbildung, Berufsausbildung, Berufstätigkeit) wurden mit einem selbstentwickelten Fragebogen erhoben. Weiterhin wurden die Patienten nach Abschluss der HBO-Therapie gefragt, wie sie die Veränderung ihres Zustands einschätzten, mit den folgenden Antwortmöglichkeiten: verschlechtert (1); unverändert (2); wenig verbessert (3); verbessert (4); sehr stark verbessert (5).

\section{Kontrollgruppe der Allgemeinbevölkerung}

Als Kontrollgruppe diente eine Teilstichprobe aus der deutschen Allgemeinbevölkerung. Im Jahr 1998 wurde eine bevölkerungsrepräsentative Stichprobe $(n=2037)$ mit den 3 Fragebögen HADS [35], MFI [36] und EORTC QLQ-C30 [37] untersucht. Um die Kontrollgruppe hinsichtlich Alter und Geschlecht der Untersuchungsgruppe der Tinnituspatienten anzupassen, wurde aus dieser Stichprobe eine Teilstichprobe so gebildet, dass mittleres Alter und Geschlechterverteilung der Patientengruppe gut entsprachen. Dies führte auf eine Stichprobengröße von $n=1699$ mit 63\% Männern und einem mittleren Alter von 44,9 Jahren.

\section{Statistische Auswertung}

Für die Datenauswertung kam die Software SPSS, Version 15.0 zur Anwendung. Die Beschreibung der Stichprobe hinsichtlich soziodemografischer und klinischer Daten erfolgte mittels deskriptiver Statistik. Generell wurde ein $\mathrm{p}<0,05$ als statistisch bedeutsam interpretiert. Zur Ermittlung von Zusammenhängen wurde der Korrelationskoeffizient nach Pearson berechnet. Mittels t-Test für unabhängige Stichproben wurden Mittelwertvergleiche zwischen Patientengruppe und Kontrollgruppe durchgeführt, die Prüfung von Mittelwertvergleichen im Verlauf erfolgte über den t-Test für abhängige Stichproben. Effektstärken zur Charakterisierung von Mittelwertunterschieden wurden gemäß Cohen [38] berechnet.

\section{Ergebnisse}

120 Tinnituspatienten mit Indikation für eine hyperbare Sauerstofftherapie nahmen unselektiert an der Studie teil. Die wichtigsten soziodemografischen Merkmale sind in $\bullet$ Tab. 1 aufgeführt. Von den 120 Patienten waren 63\% männlich, das Durchschnittsalter betrug 44,8 Jahre (SD 11,5; Range: 14-76 Jahre). Gemäß der aktuellen Tinnitus-Leitlinie von 2010 war bei 55 Patienten ein akuter Tinnitus (bis 3 Monate Symptomdauer) und bei 65 Patienten ein chronischer Tinnitus (4-12 Monate Symptomdauer) zu verzeichnen. Hinsichtlich des Hörvermögens zu 
Tab. 2 Mittelwertverläufe bei den Patienten (t1-t3) und Vergleich zur Allgemeinbevölkerung (KG).

\begin{tabular}{|c|c|c|c|c|c|c|c|c|c|c|c|}
\hline & \multicolumn{2}{|c|}{$\begin{array}{c}t 1 \\
n=120\end{array}$} & \multicolumn{2}{|c|}{$\begin{array}{c}t 2 \\
n=120\end{array}$} & \multicolumn{2}{|c|}{$\begin{array}{c}t 3 \\
n=98\end{array}$} & \multicolumn{2}{|c|}{$\begin{array}{c}\text { Allg.-Bev. } \\
n=1699\end{array}$} & \multirow[t]{2}{*}{$\begin{array}{c}\text { ES } \\
\text { t1-KG }\end{array}$} & $\begin{array}{l}\text { t-Test } \\
\text { t1-t2 }\end{array}$ & \\
\hline & M & SD & M & SD & M & SD & $\mathbf{M}$ & SD & & $\mathbf{t}$ & \\
\hline \multicolumn{12}{|l|}{ HADS } \\
\hline Angst & 7,87 & 3,72 & 6,60 & 3,35 & 6,63 & 3,37 & 4,70 & 3,25 & 0,98 & 5,47 & $* * *$ \\
\hline Depressivität & 6,33 & 3,34 & 5,48 & 3,24 & 5,71 & 3,48 & 4,36 & 3,70 & 0,53 & 3,50 & *** \\
\hline \multicolumn{12}{|l|}{ MFI (Fatigue) } \\
\hline allgemeine Fatigue & 11,34 & 3,43 & 10,05 & 3,21 & 9,88 & 3,48 & 8,31 & 3,51 & 0,86 & 4,47 & $* * *$ \\
\hline körperliche Fatigue & 10,94 & 3,41 & 9,86 & 3,66 & 10,06 & 3,61 & 7,89 & 3,83 & 0,80 & 3,94 & $* * *$ \\
\hline reduzierte Aktivität & 9,48 & 3,42 & 8,81 & 3,23 & 8,73 & 3,40 & 7,90 & 3,58 & 0,44 & 2,72 & ** \\
\hline reduzierte Motivation & 9,22 & 2,87 & 8,62 & 2,81 & 8,61 & 2,88 & 7,66 & 3,19 & 0,49 & 2,62 & ** \\
\hline mentale Fatigue & 10,49 & 3,97 & 9,73 & 3,45 & 8,95 & 3,55 & 7,51 & 3,23 & 0,92 & 2,75 & ** \\
\hline \multicolumn{12}{|l|}{ EORTC QLQ } \\
\hline körperl. Funkt. & 81,22 & 22,24 & 84,56 & 17,82 & 84,90 & 20,23 & 92,33 & 14,38 & $-0,77$ & 3,19 & $* *$ \\
\hline Rollen-Funkt. & 60,28 & 30,42 & 63,89 & 29,89 & 64,63 & 29,72 & 89,94 & 20,96 & $-1,42$ & 1,68 & \\
\hline emotionale Funkt. & 48,47 & 24,23 & 63,89 & 24,62 & 62,93 & 23,45 & 78,52 & 21,09 & $-1,42$ & 7,27 & $* * *$ \\
\hline kognitive Funkt. & 69,17 & 25,44 & 77,36 & 22,85 & 77,38 & 23,48 & 92,54 & 15,72 & $-1,49$ & 4,38 & $* * *$ \\
\hline soziale Funkt. & 62,36 & 31,64 & 70,56 & 28,89 & 71,77 & 30,87 & 92,27 & 18,05 & $-1,66$ & 3,93 & $* * *$ \\
\hline globale Lebensqualität & 50,56 & 17,87 & 57,57 & 17,45 & 60,37 & 19,07 & 72,52 & 21,74 & $-1,01$ & 4,42 & $* * *$ \\
\hline Fatigue & 42,69 & 22,59 & 38,06 & 20,81 & 33,56 & 19,14 & 15,30 & 20,88 & 1,31 & 2,28 & $*$ \\
\hline Übelkeit/Erbrechen & 9,31 & 21,48 & 6,39 & 13,87 & 3,40 & 9,86 & 2,75 & 9,97 & 0,66 & 1,53 & \\
\hline Schmerz & 40,00 & 29,81 & 31,39 & 28,19 & 29,59 & 25,50 & 13,18 & 22,78 & 1,18 & 3,95 & $* * *$ \\
\hline Dyspnoe & 15,00 & 21,97 & 9,44 & 18,93 & 9,52 & 16,58 & 6,22 & 17,98 & 0,49 & 3,29 & $* * *$ \\
\hline Schlafprobleme & 51,94 & 36,87 & 45,83 & 30,88 & 36,39 & 29,92 & 14,73 & 26,00 & 1,43 & 2,28 & * \\
\hline Appetitverlust & 12,50 & 21,63 & 11,94 & 19,71 & 8,84 & 16,27 & 4,75 & 15,25 & 0,51 & 0,23 & \\
\hline Verstopfung & 7,50 & 20,48 & 4,44 & 14,93 & 5,10 & 18,13 & 3,20 & 12,93 & 0,33 & 1,62 & \\
\hline Durchfall & 10,28 & 18,73 & 5,28 & 13,66 & 4,76 & 12,66 & 2,86 & 11,65 & 0,64 & 2,86 & ** \\
\hline finanzielle Schwierigk & 36,67 & 35,21 & 28,89 & 35,09 & 31,63 & 32,94 & 5,06 & 17,18 & 1,84 & 3,35 & $* * *$ \\
\hline
\end{tabular}

Beginn der Therapie (t1) gaben 26 (21,7\%) Patienten ein normales Hörvermögen, 73 Patienten (60,8\%) ein leicht eingeschränktes Hörvermögen und 21 Patienten (17,5\%) ein stark eingeschränktes Hörvermögen an.

\section{Vergleich der Patienten (zu t1) mit der Allgemeinbevölkerung}

- Tab. 2 fasst zunächst die Mittelwertverläufe für die 3 Fragebögen und die 3 Messzeitpunkte zusammen. In allen Bereichen der gesundheitsbezogenen Lebensqualität, Fatigue, Angst und Depressivität haben die Patienten zu t1 signifikant $(p<0,001)$ ungünstigere Werte als die Allgemeinbevölkerung ( $\bullet$ Tab. 2).

Bezüglich der gesundheitsbezogenen Lebensqualität (EORTC QLQC30) sind die Unterschiede zwischen Patienten und Allgemeinbevölkerung in folgenden Bereichen am stärksten ausgeprägt (höchste Effektstärken): Finanzielle Schwierigkeiten $(\mathrm{d}=1,84)$, Soziale Funktionsfähigkeit $(d=1,66)$, Kognitive Funktionsfähigkeit $(d=1,49)$, Schlafprobleme $(\mathrm{d}=1,43)$, Rollen-Funktionsfähigkeit $(\mathrm{d}=1,42)$ und Emotionale Funktionsfähigkeit $(\mathrm{d}=1,42)$. Die Einzelsymptome (Übelkeit/Erbrechen, Kurzatmigkeit, Appetitverlust, Verstopfung, Durchfall) sind ebenfalls stärker ausgeprägt bei den Tinnituspatienten, jedoch sind die Effektstärken mit Werten um 0,50 geringer.

Hinsichtlich Fatigue dominiert die Skala Mentale Fatigue $(d=0,92)$, wogegen die Skalen Reduzierte Aktivität und Reduzierte Motivation geringere Einbußen aufweisen. Angst unterliegt einem stärkeren Effekt $(d=0,98)$ als Depressivität $(d=0,53)$.

Verläufe der Lebensqualität bei den Patienten

In allen Skalen zeigt sich von t1 nach t2 eine Verbesserung ( $\bullet$ Tab. 2). In der Folgezeit (Verlauf von t2 bis t3) bleiben die Werte in den meisten Skalen relativ konstant. 0 Abb. 1 veran- schaulicht auf der Ebene der Summenwerte die Trends. Die meisten Verbesserungen finden sich in denjenigen Variablen, bei denen am Beginn die größten Defizite (größte Abstände zu den Werten der Allgemeinbevölkerung) zu verzeichnen waren.

Vergleicht man die Werte am Untersuchungsende ( $\mathrm{t} 3$ ) mit denen der Allgemeinbevölkerung, so bleiben noch immer deutliche Unterschiede bestehen, insbesondere in den folgenden Bereichen: Finanzielle Schwierigkeiten, Rollen-Funktionsfähigkeit, Soziale Funktionsfähigkeit, Kognitive Funktionsfähigkeit, Fatigue (EORTC QLQ-C30) und Schlafprobleme. Bei den meisten der Variablen wird der zu t1 feststellbare Abstand zur Allgemeinbevölkerung bis zum Zeitpunkt $\mathrm{t} 3$ um etwa die Hälfte verringert, vgl. auch $\bullet$ Abb. 1.

\section{Abhängigkeit von Geschlecht, Alter, Reha-Teilnahme und Symptomdauer}

Frauen weisen im Vergleich zu Männern in 8 der 22 Variablen signifikant ungünstigere Werte zu t1 auf; insbesondere hinsichtlich Fatigue, die anderen Mittelwertunterschiede sind nicht signifikant (vgl. @ Tab. 3). Das Alter hat nur einen geringen Einfluss auf die Lebensqualität zu t1. Ebenso zeigen sich zwischen den akuten und chronischen Patienten (gemäß neuer Leitlinie) keinerlei signifikante Mittelwertunterschiede. Patienten, die die HBO im Rahmen einer Reha-Maßnahme erhalten, haben eine geringer ausgeprägte körperliche und Rollen-Funktionalität sowie mehr Schmerzen.

\section{Verbesserung von $\mathbf{t} 1$ nach $\mathbf{t} 2$ mittels direkter Einschätzung}

Auf der 5-stufigen Skala bezüglich der Veränderung im Verlauf der ersten beiden Wochen ergaben sich die folgenden relativen 

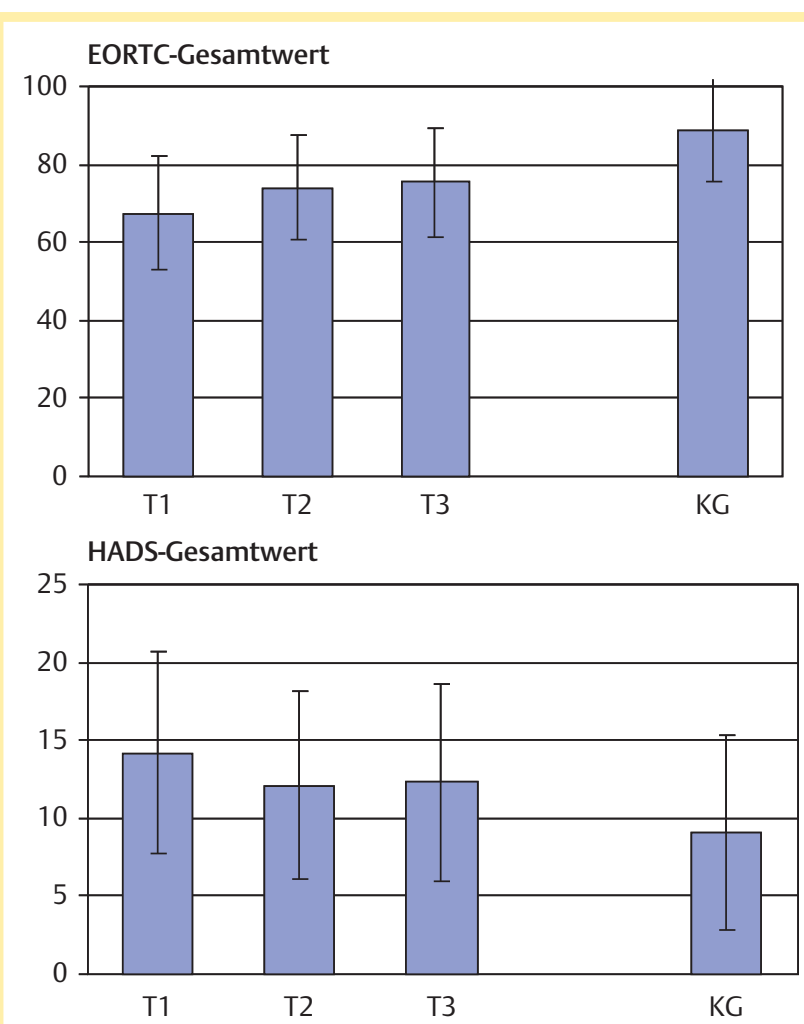

MFI-Gesamtwert

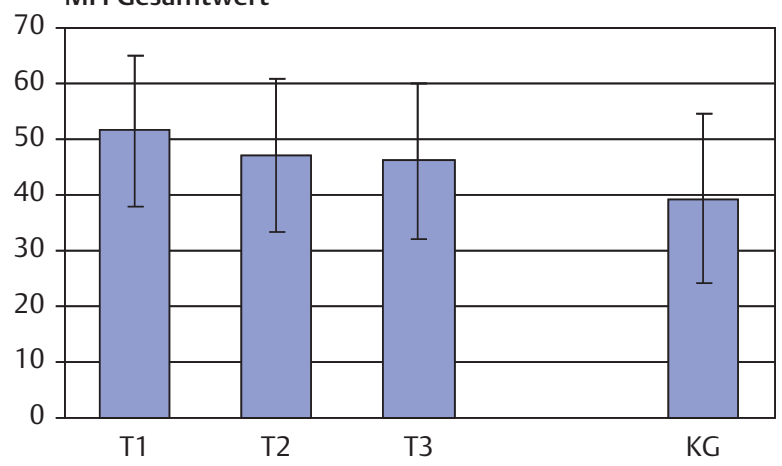

Abb. 1 Mittelwertverläufe für die Gesamtwerte von EORTC QLQ-C30, HADS und MFI. T1, T2, T3: Patienten; KG: Kontrollgruppe (Allgemeinbevölkerung).

Häufigkeiten: verschlechtert (2,5\%), unverändert (18,3\%), wenig verbessert (18,3\%), verbessert (45,8\%) und sehr stark verbessert (15,0\%). Der Mittelwert beträgt $\mathrm{M}=3,53$ ( $S D=1,04)$.

Zusammenhänge zwischen dieser Veränderungs-Einschätzung und den Differenzen aus den Fragebogenskalen werden in $\bullet$ Tab. 3 auf 2 Arten angegeben. Zum einen werden die VeränderungsAngaben mit den Werten zu t2 korreliert, zum anderen mit den Verbesserungen von $\mathrm{t} 1$ nach $\mathrm{t} 2$ (Differenz zwischen $\mathrm{t} 2$ - und $\mathrm{t} 1$ Werten).

Die Korrelationen sind insgesamt gering. Korrelationen gibt es zur Angst, Depressivität und Fatigue (MFI) in einer Größenordnung von rund $-0,20$ : Wer Verbesserungen angibt, ist zu t2 auch weniger ängstlich, weniger depressiv und hat ein geringeres Fatigue-Ausmaß. Die Beziehungen zu den ebenfalls in $\bullet$ Tab. 3 angegebenen Differenzwerten t2-t1 sind insgesamt sehr schwach, nur ein Koeffizient übersteigt den Wert von 0,20.

\section{Diskussion}

$\nabla$

Tinnituspatienten sind bezüglich der gesundheitsbezogenen Lebensqualität in allen Bereichen signifikant eingeschränkt. Besonders ausgeprägt ist dies für die psychosozialen Bereiche mit Effektstärken um 1,50. Der Begriff der Effektstärke ist hier rein statistisch zu verstehen als der an der Standardabweichung relativierte Mittelwertunterschied [34], ohne dass kausale Effekte unterstellt werden. Soziale Funktionen sind im Vergleich zur Allgemeinbevölkerung am stärksten reduziert, kognitive und emotionale Funktionalität sind ebenfalls sehr stark betroffen. Die körperliche Funktionsfähigkeit ist ebenfalls eingeschränkt $(d=0,77)$, aber nicht so massiv wie die psychosozialen Aspekte. Dies entspricht auch weitgehend den Befunden, die mit dem SF36 erhoben wurden $[20,21]$. Dabei ist zu beachten, dass es sich bei der Allgemeinbevölkerung durchaus nicht nur um gesunde Personen handelt, sondern dass auch hier Personen mit chronischen Erkrankungen vertreten sind.

Die zu t1 erfassten HADS-Werte für Angst und Depressivität $(7,9 / 6,3)$ liegen in der gleichen Größenordnung wie die HADSMittelwerte, welche in anderen Studien bei Tinnnituspatienten ermittelt wurden: Bartels et al. [39]: (8,0/7,3); Cima et al. [40] $(6,4 / 7,6)$. Daraus lässt sich schlussfolgern, dass die Patienten in der vorliegenden Studie im Mittel von ähnlichem Schweregrad betroffen waren. Vergleicht man die Belastung der Tinnituspatienten (zu t1) mit jener von Krebspatienten während der Akutphase im Krankenhaus [41] oder mit Herz-Kreislauf-Patienten [30], so sind die Belastungswerte bei den Tinnituspatienten generell sogar noch höher.

Im Laufe der Zeit bilden sich die Einschränkungen deutlich zurück. Auch andere Studien berichten von einer Verbesserung der Lebensqualität nach Therapie. In einer umfangreichen Studie aus Österreich mit 360 Tinnituspatienten [29] berichteten nach HBO-Therapie $49 \%$ von einer Verbesserung, $44 \%$ von einer gleichbleibenden Situation und 7\% von einer Verschlechterung; ein Vergleich von Prä- und Postwerten mit standardisierten Fragebögen fand in dieser Untersuchung aber nicht statt. Ein interessanter Befund dieser Studie war jedoch, dass die zuvor erfragte Erwartung, ob die HBO-Therapie helfen werde, mit dem subjektiv eingeschätzten Outcome positiv korrelierte. In einer Studie mit lediglich 16 Tinnituspatienten [42] berichteten 6 von einer subjektiven Verbesserung, 8 bemerkten keine Veränderung, und 2 eine Verschlechterung, wobei die Gesamtanalyse auf Mittelwertebene aber nur sehr geringe Änderungen erbrachte. Hierbei ist zu berücksichtigen, dass es sich in dieser Studie um Fälle mit schwerem Tinnitus handelte, die in vorhergehenden Therapien nicht erfolgreich behandelt werden konnten.

Da keine Kontrollgruppe ohne hyperbare Sauerstofftherapie untersucht wurde, kann hier nicht geklärt werden, welcher Teil der Verbesserung innerhalb der ersten 2 Wochen auf die Therapie und welcher auf Spontanremission oder auf andere Maßnahmen im Rahmen der Reha rückführbar ist. Damit soll die Studie in keiner Weise als Evaluationsinstrument dieser Therapie verstanden werden. Ein Vergleich mit einer Gruppe ohne diese Therapie wäre jedoch auch problematisch gewesen, da durchaus anzunehmen ist, dass die Intervention eher bei schwerer betroffenen Patienten durchgeführt worden wäre, sodass die Ausgangslage auch nicht vergleichbar wäre. Eine randomisierte $\mathrm{Zu}-$ weisung der Patienten in 2 Untersuchungsarme mit bzw. ohne hyperbare Sauerstofftherapie hätte dies Problem beseitigen können, war jedoch im vorliegenden Fall nicht möglich. 


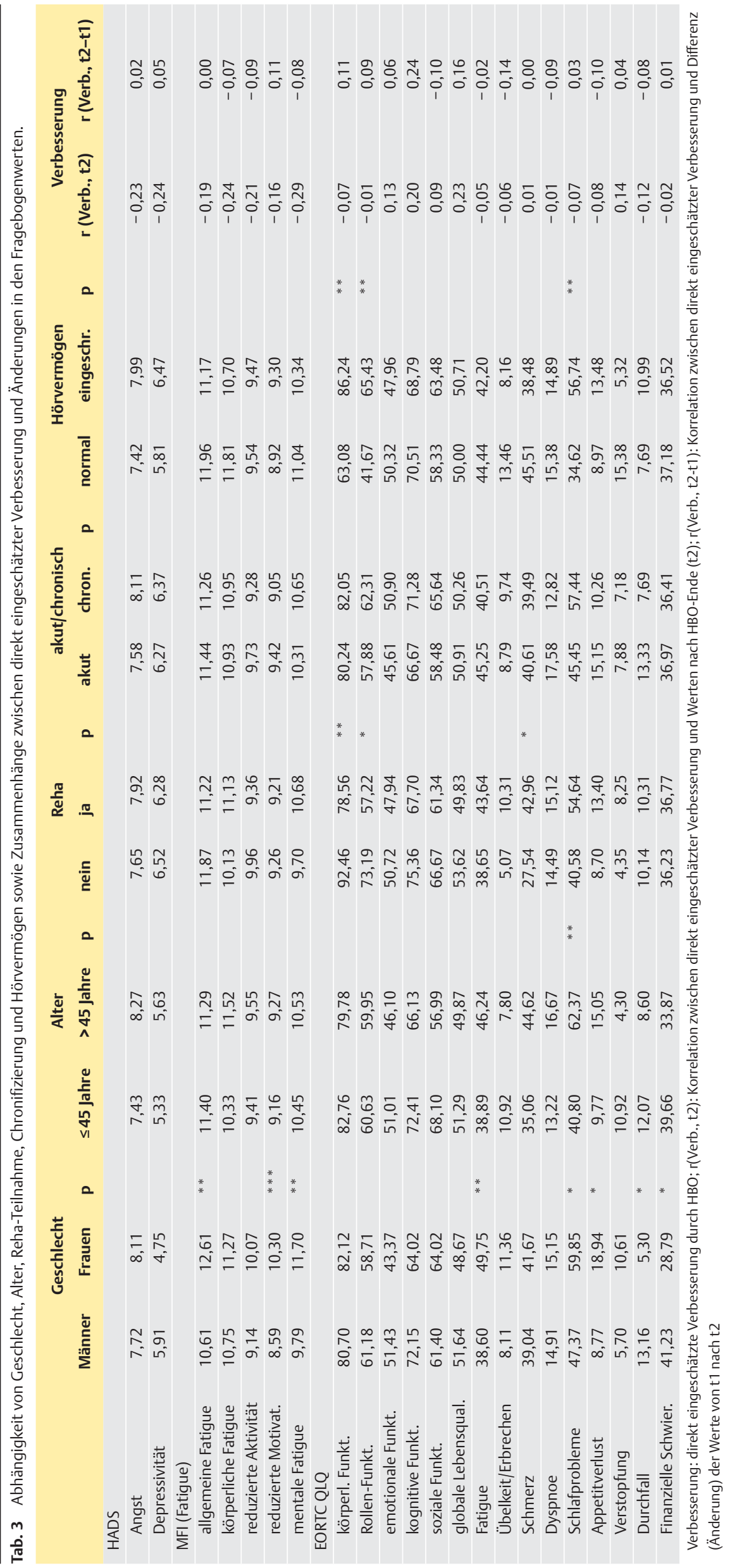


Wesentlich ist, dass nach Untersuchungsende, also 4 Wochen nach Ende der Sauerstofftherapie, noch immer erhebliche Abstände zur Allgemeinbevölkerung bestehen bleiben. Die Gesamteinschätzung der globalen Lebensqualität im EORTC QLQC30 steigert sich zwar von rund $50 \mathrm{zu}$ t1 auf rund $60 \mathrm{zu}$ t3, die Allgemeinbevölkerung liegt aber bei rund 70. Generell kann man zusammenfassen, dass sich in den meisten Variablen der Abstand zwischen den Patienten und den Werten der Allgemeinbevölkerung im Laufe der Untersuchungszeit (von t1 bis t3) etwa halbiert hat, was auch $\bullet$ Abb. 1 illustriert.

Die Skala Finanzielle Schwierigkeiten zeigt den größten Mittelwertunterschied zwischen den Patienten und der Allgemeinbevölkerung. Da der Fragebogen für Patienten konzipiert wurde und die Frage generell so verstanden wird, dass sie auf erkrankungsbezogene finanzielle Schwierigkeiten abzielt, ist der Vergleichswert der Allgemeinbevölkerung hier nur eingeschränkt interpretierbar. Dennoch verweist die hohe Effektstärke darauf, dass sich die Patienten durch die Erkrankung auch finanziell belastet fühlen.

Die differenzierte Einschätzung von Fatigue mit dem MFI zeigte in den Bereichen Mentale Fatigue, Allgemeine Fatigue und Körperliche Fatigue die massivsten Einschränkungen bei den Patienten, wogegen die Dimensionen Reduzierte Aktivität und Reduzierte Motivation weniger betroffen waren. Die deutlichsten Verbesserungen von $\mathrm{t} 1$ nach $\mathrm{t} 2$ zeigten sich bei Allgemeiner und Körperlicher Fatigue; die klarste Beziehung zwischen der direkt eingeschätzten Verbesserung durch die Therapie und den Fatigue-Facetten ergab sich für die Mentale Fatigue. Es lohnt damit durchaus, die verschiedenen Dimensionen von Fatigue differenziert zu betrachten.

Patientinnen sind stärker belastet als Patienten, insbesondere im Bereich Fatigue. Da jedoch auch in der Allgemeinbevölkerung Frauen im Mittel etwas höhere Fatigue-Werte angeben als Männer [36], ist nur ein Teil des Geschlechtsunterschiedes der Patienten auf die unterschiedliche Wirkung des Tinnitus zurückzuführen; es bleibt jedoch auch nach Berücksichtigung dieses Faktors in allen Fatigue-Skalen eine stärkere Belastung der Frauen. Das Alter hat dagegen kaum Einfluss auf die Belastung durch Tinnitus.

In dieser Studie wurde darauf verzichtet, die Beziehungen zwischen Angst, Depressivität, Fatigue und Lebensqualität zu analysieren. In der Literatur werden Modelle berichtet, welche die Wirkung von Persönlichkeitseigenschaften (Typ D) auf die Lebensqualität, mediiert durch Angst und Depressivität, beschreiben [39] oder auch Analysen der Effekte von Angst und Depressivität auf die Lebensqualität [43]. Da es einen großen Bereich von Konstruktüberlappung zwischen Depressivität, Fatigue und Lebensqualität gibt, erscheinen jedoch solche kausalen Zusammenhangsinterpretationen (psychische Belastung bewirke Einschränkungen der Lebensqualität) nicht angemessen.

Bei der Interpretation der Veränderungen im Laufe der Therapie ist zu beachten, dass sich die Bewertungsmaßstäbe der Patienten im Sinne des Response-Shift-Konzepts [44] geändert haben können. Sollte dies der Fall sein (was hier nicht geprüft werden kann), so sind die „wahren“ Veränderungen noch höher einzuschätzen als die hier vorgestellten Mittelwertverschiebungen. Die subjektive Bewertung der Verbesserung durch die Therapie führte auf einen Mittelwert von 3,5 bei einer 5-stufigen Skala (Bereich 1-5), mit einer Häufung der Kategorie „verbessert“. Die direkt abgefragte subjektive Bewertung der Veränderung korreliert jedoch nur geringfügig mit der indirekten Erfassung, welche sich aus den Differenzen zwischen Post- und Prä-Werten der standardisierten Fragebögen ergibt. Bei Studien, die sich auf die direkte Abfrage der Veränderung beschränken, ist also Vorsicht geboten, ob diese Änderungen sich auch in Mittelwertunterschieden von Skalen etablierter Fragebögen widerspiegeln würden. Möglicherweise sind die Urteile zur subjektiv eingeschätzten Verbesserung anfälliger für Effekte sozialer Erwünschtheit als die Selbsturteile zur Lebensqualität und die aus den Differenzen gewonnenen Veränderungswerte.

Einige Limitationen der Studie seien kurz genannt. Es wurde bereits darauf hingewiesen, dass die Veränderungen von t1 zu t2 nicht als Wirkungen der HBO-Therapie interpretiert werden sollten, da die Effekte nicht von Spontanremissionen oder den anderen Reha-Maßnahmen getrennt werden können. Die Patientengruppe hatte als gemeinsames Merkmal, dass eine Indikation für eine HBO-Therapie gegeben war; damit sind leichtere Formen von Tinnitus nicht oder unterproportional vertreten. Der Stichprobenumfang ist für die Analyse der Gesamtgruppe (Verläufe sowie Vergleiche zur Allgemeinbevölkerung) ausreichend, für Binnenvergleiche (z.B. mit vs. ohne Reha) gelangt der Stichprobenumfang an seine Grenzen. Die Nutzung der Fragebögen EORTC QLQ-C30 und MFI gestattet zwar eine ungewöhnlich differenzierte Analyse von Fatigue und Lebensqualität, jedoch sind die Ergebnisse mit Studien, welche den SF-36 nutzen, dadurch nicht mehr direkt vergleichbar. Es wurden keine tinnitusspezifischen Fragebögen eingesetzt. Damit ist das Belastungsprofil nicht auf diese Patientengruppe zugeschnitten, der Vorteil der eingesetzten störungsübergreifenden Fragebögen besteht aber darin, dass Vergleiche zur Allgemeinbevölkerung möglich sind.

\section{Abstract}

\section{Mental Distress and Quality of Life in Tinnitus Patients \\ $\nabla$}

Background: Tinnitus is a disease with a high prevalence that is often combined with psychiatric comorbidity. The aim of this study was to identify the dimensions of quality of life in which tinnitus patients are especially affected, and how these affections change during a therapy, including a hyperbaric oxygen therapy.

Material and Methods: 120 patients suffering from tinnitus were examined at 3 time points: at the beginning (t1) and the end (t2) of a 2-week hyperbaric oxygen therapy, and 4 weeks later ( $\mathrm{t} 3$ ). The following questionnaires were adopted: Hospital Anxiety and Depression Scale, Multidimensional Fatigue Inventory, and the quality of life instrument EORTC QLQ-C30.

Results: Compared with the general population, tinnitus patients were impaired in all areas of quality of life. The greatest differences were found in the scales Social, Cognitive, Emotional and Role Functioning and in the field of financial difficulties with effect sizes of about 1.5. During the therapy, the scores improved, reaching roughly the middle between the initial patients' scores and the values of the general population. The directly assessed subjective improvement due to the therapy was only marginally correlated with the differences in the questionnaires. Conclusion: Since there was no control group without hyperbaric oxygen therapy, the results do not justify conclusions about the effectiveness of this therapy. However, the findings document multiple impairments of the patients (especially psycho-social disturbances) and show hints for supportive offers. 
Interessenkonflikt: Die Autoren geben an, dass kein Interessenkonflikt besteht.

\section{Literatur}

1 Hesse G. Tinnitus. Stuttgart: Thieme, 2008

2 Kempf HG, Zenner HP. Tinnitus. In: Zenner HP, Hrsg. Praktische Therapie von HNO-Krankheiten. Stuttgart: Schattauer, 2008; 133-141

3 Pilgramm M, Rychlik R, Lebisch H, Siedentop H, Goebel G, Kirchhoff D. Tinnitus in der Bundesrepublik Deutschland - Eine repräsentative epidemiologische Studie. HNO aktuell 1999; 7: 261-265

4 Tönnies S. Leben mit Ohrgeräuschen. Selbsthilfe bei Tinnitus. Heidelberg: Asanger, 2001

5 Streppel M, Walger M, von Wedel H, Wedel H, Gaber E. Hörstörungen und Tinnitus. Gesundheitsberichterstattung des Bundes, Heft 29. Berlin: Robert Koch Institut, 2006

6 Nondahl DM, Cruickshanks KJ, Dalton DS, Klein BEK, Klein R, Schubert $C R$, Tweed $T S$, Wiley $T L$, The impact of tinnitus on quality of life in older adults. J Am Acad Audiol 2007; 18: 257-266

7 Holmes S, Padgham ND. Review paper: more than ringing in the ears: a review of tinnitus and its psychosocial impact. J Clin Nursing 2009; 18: 2927-2937

8 Greimel KV, Biesinger E. Psychologische Prinzipien bei der Behandlung von Tinnituspatienten. HNO 1999; 47: 130-134

9 Goebel G. Somatoforme Störung bei Tinnitus. Psychiatr Danubina 2000; 12: 215-228

10 Zirke N, Goebel G, Mazurek B. Tinnitus and psychological comorbidities. HNO 2010; 58: 726-732

11 Schaaf H, Dölberg D, Seling B, Märtner M. Komorbidität von Tinnituserkrankungen und psychiatrischen Störungen. Nervenarzt 2003; 74: 72-75

12 Goebel G, Fichter M. Psychiatrische Komorbidität bei Tinnitus. In: Biesinger E, Iro H, Hrsg. HNO Praxis heute: Tinnitus. Heidelberg: Springer, 2005; $137-150$

13 Jacobi F, Wittchen HU, Holting C, Hofler M, Pfister H, Muller N, Lieb R, Prevalence, co-morbidity and correlates of mental disorders in the general population: results from the German Health Interview and Examination Survey (GHS). Psychol Med 2004; 34: 597-611

14 Stobik C, Weber RK, Munte TF, Walter M, Frommer A, Evidence of psychosomatic influences in compensated and decompensated tinnitus. Int J Audiol 2005; 44: 370-378

15 Zoger S, Svedlund J, Holgers KM. Relationship between tinnitus severity and psychiatric disorders. Psychosomatics 2006; 47: 282-288

16 Belli S, Belli H, Bahcebasi T, Ozcetin A, Alpay E, Ertem U. Assessment of psychopathological aspects and psychiatric comorbidities in patient affected by tinnitus. Eur Arch Otorhinolaryngol 2008; 265: 279-285

17 Konzag TA, Rubler D, Bandemer-Greulich U, Frommer J, Fikentscher E. Tinnitusbelastung und psychische Komorbidität bei ambulanten subakuten und chronischen Tinnituspatienten. Z Psychosom Med Psychother 2005; 51: 247-260

18 Graul J, Klinger R, Greimel KV, , Rustenbach S, Nutzinger DO, Differential outcome of a multimodal cognitive-behavioral inpatient treatment for patients with chronic decompensated tinnitus. Int Tinnitus J 2008 14: 73-81

19 Kamalski DM, Hoekstra CE, van Zanten BG, Grolman W, Rovers MM. Measuring disease-specific health-related quality of life to evaluate treatment outcomes in tinnitus patients: A systematic review. Otolaryngology - Head Neck Surgery 2010; 143: 181-185

20 Davis AC, Roberts $H$. Tinnitus and health status: SF-36 profile and accident prevalence. In: Reich GE, Vernon JA (eds.). Proceedings of the Fifth International Tinnitus Seminar. Portland, OR: American Tinnitus Association, 1995; 257-265

21 Wilson C, Lewis P, Stephens D. The short form 36 (SF36) in a specialist tinnitus clinic. Int J Audiol 2002; 41: 216-220

22 Lenarz T. Epidemiologie. In: Feldmann H. Tinnitus: Grundlagen einer rationalen Diagnostik und Therapie. Stuttgart: Thieme, 1998; 77-83

23 Erlandsson SI, Hallberg IR-M. Prediction of quality of life in patients with tinnitus. Br J Audiol 2000; 34: 11-20
24 AWMF-Leitlinie Tinnitus. http://www.awmf.org/uploads/tx_szleitlinien/ 017-064I_S1_Tinnitus.pdf

25 Kuokkanen J, Aarnisalo AA, Ylikoski J. Efficiency of hyperbaric oxygen therapy in experimental acute acoustic trauma from firearms. Acta Otolaryngol Suppl 2000; 543: 132-134

26 Schumann K, Lamm K, Hettich M. Zur Wirksamkeit der hyperbaren Sauerstofftherapie bei alten Hörstörungen. Bericht über 557 Fälle aus dem Jahre 1989. HNO 1990; 38: 408-411

27 Ganström G, Axelsson A, Fornander J. Effects of hyperbaric oxygen on tinnitus levels in chronic audiologic diseases. Proceedings from the XVII $^{\text {th }}$ EUBS, Heraklion, Greece $1991 ; 157-163$

28 Bennett $M$, Kertesz T, Yeung P. Hyperbaric oxygen therapy for idiopathic sudden sensorineural hearing loss and tinnitus: a systematic review of randomized controlled trials. J Laryngol Otol 2005; 119: 791-798

29 Porubsky C, Stiegler P, Matzi V, Lipp C, Kontaxis A, Klemen H, Walch C, Smolle-Jüttner $F$. Hyperbaric oxygen in tinnitus: Influence of psychosocial factors on treatment results? ORL - Oto-Rhino-Laryngology 2007; 69: 107-112

30 Herrmann $C$, Buss $U$, Snaith RP. HADS-D Hospital Anxiety and Depression Scale - Deutsche Version. Ein Fragebogen zur Erfassung von Angst und Depressivität in der somatischen Medizin. Testdokumentation und Handanweisung. Bern: Huber, 1995

31 Bjelland I, Dahl AA, Haug TT, Neckelmann D. The validity of the Hospital Anxiety and Depression Scale. An updated literature review. J Psychosom Res 2002; 52: 69-77

32 Smets EMA, Garssen B, Bonke B, De Haes JCJM. The multidimensional fatigue inventory (MFI): Psychometric qualities of an instrument to assess fatigue. J Psychosom Res 1995; 39: 315-325

33 Aaronson NK, Ahmedzai S, Bergman B, Bullinger M, Cull A, Duez NJ, Filiberti A, Flechtner $H$, Fleishman SB, Dehaes JCJM, Kaasa S, Klee $M$, Osoba D, Razavi D, Rofe PB, Schraub S, Sneeuw M, Takeda F. The European Organisation for Research and Treatment of Cancer QLQC30: A quality-of-life instrument for use in international clinical trials in oncology. J Natl Cancer Inst 1993; 85: 365-376

34 Hinz A, Singer S, Stolzenburg J-U, Höckel M, , Stolzenburg J-U, Kortmann $R-D$, Finck Barboza $C$, Is it useful to calculate sum scores of the EORTC QLQ-C30? Support Care Cancer 2011; 19 (Suppl): 202-203

35 Hinz A, Brähler E. Normative values for the Hospital Anxiety and Depression Scale (HADS) in the general German population. J Psychosom Res 2011; 71: 74-78

36 Schwarz $R$, Krauss $O$, Hinz A. Fatigue in the general population. Onkologie 2003; 26: 140-144

37 Schwarz R, Hinz A. Reference data for the quality of life questionnaire EORTC QLQ-C30 in the general German population. Eur J Cancer 2001; 37: 1345-1351

38 Cohen J. A power primer. Psychol Bull 1992; 112: 155-159

39 Bartels H, Pedersen SS, van der Laan BFAM, Stall MJ, Albers FWJ, Middel $B$, The impact of Type D personality on health-related quality of life in tinnitus patients is mainly mediated by anxiety and depression. Otology and Neurotology 2010; 31: 11-18

40 Cima RFF, Crombez G, Vlaeyen JWS. Catastrophing and fear of tinnitus predict quality of life in patients with chronic tinnitus. Ear and Hearing 2011; 32: 634-641

41 Hinz A, Krauss $O$, Hauss JP, Höckel $M$, Kortmann RD, Stolzenburg $J-U$, Schwarz $R$, Anxiety and depression in cancer patients compared with the general population. Eur J Cancer Care 2010; 19: 522-529

42 Tan J, Tange RA, Dreschler WA, von der Kleij A, Tromp EC. Long-term effect of hyperbaric oxygenation treatment on chronic distressing tinnitus. Scand Audiol 1999; 28: 91-96

43 Bartels H, Middel BL, van der Laan BFAM, Staal MJ, Albers FWJ. The additive effect of co-occuring anxiety and depression on health status, quality of life and coping strategies in help-seeking tinnitus sufferers. Ear Hearing 2008; 29: 947-956

44 Sprangers MAG, Schwartz CE. Integrating response shift into healthrelated quality of life research: A theoretical model. Soc Sci Med 1999; 48: 1507-1515 



\title{
4. Zusammenfassung
}

Dissertation zur Erlangung des akademischen Grades Dr. med.

\section{Psychische Belastung und Lebensqualität bei Tinnituspatienten}

\author{
eingereicht von: Steffi Hesse \\ angefertigt an: Abteilung für Medizinische Psychologie und Medizinische \\ Soziologie der Universität Leipzig \\ betreut von: $\quad$ Prof. Dr. rer. nat. habil. A. Hinz \\ Dr. rer. med. A. Meyer
}

März 2013

Tinnitus ist eine akustische Wahrnehmung des Menschen, die zusätzlich zum Schall, der auf das Ohr wirkt, wahrgenommen wird. Es ist ein Höreindruck (Ohrgeräusch, Ohrenklingen, Ohrensausen), der nicht auf der Stimulation durch einen äußeren Schallreiz beruht. Jährlich erleben in Deutschland rund zehn Millionen Menschen in irgendeiner Form einen akuten Tinnitus. Es wird geschätzt, dass ca. drei Millionen Personen von chronischem Tinnitus betroffen sind. Das entspricht vier Prozent der Gesamtbevölkerung. 1,5 Millionen Bundesbürger haben einen mittelschwer bis schwer ausgeprägten Tinnitus.

Tinnitus führt durch den Verlust der Stille, durch die ständigen Geräusche und das Gefühl, ihnen nicht entrinnen zu können, bei vielen Betroffenen zu körperlichen und psychischen Problemen, die Leben und Arbeit massiv beeinträchtigen. Zwischen subjektiver Belastung durch Ohrgeräusche und psychischen Störungen wurden stark ausgeprägte Zusammenhänge nachgewiesen. Der Tinnitusschweregrad steht in Beziehung zu psychischen Störungen und zum Schweregrad von Angststörungen und Depressionen.

Neben Studien zur psychischen Belastung existieren auch Untersuchungen zur gesundheitsbezogenen Lebensqualität bei Tinnituspatienten. Über die Verläufe von psychischer Belastung und Lebensqualität bei Tinnituspatienten ist allerdings wenig bekannt. Generell handelt es sich entweder um Querschnittsstudien, welche eine Momentaufnahme der Lebensqualität der Patienten zeigen, typischerweise in einem 
Setting als Klinikpatienten, oder es wird andererseits versucht, die Wirksamkeit von Therapien nachzuweisen, dann aber i. A. ohne Bezug zu den Werten der Allgemeinbevölkerung.

Die vorliegende Arbeit verfolgt deshalb das Anliegen, die gesundheitsbezogene Lebensqualität und das psychische Befinden im Verlauf zu untersuchen und einen Vergleich mit Daten der Allgemeinbevölkerung durchzuführen.

Die konkreten Zielstellungen der vorliegenden Arbeit sind die folgenden:

- Analyse von Lebensqualität, Fatigue und psychischer Belastung bei Tinnituspatienten durch Vergleiche mit der Allgemeinbevölkerung

- Analyse von Verläufen der gesundheitsbezogenen Lebensqualität während einer hyperbaren Sauerstofftherapie über drei Messzeitpunkte sowie

- Prüfung der Wirkung soziodemografischer Variablen und Reha-Teilnahme auf die gesundheitsbezogene Lebensqualität.

Im Zeitraum von 2004-2006 wurden 120 Patienten, die im Fachklinikum Brandis an einer hyperbaren Sauerstofftherapie (HBO) wegen Tinnitus teilnahmen, hinsichtlich Angst und Depressivität, Fatigue sowie gesundheitsbezogener Lebensqualität untersucht. Eingeschlossen wurden 97 Patienten, die im Rahmen einer Rehabilitationsmaßnahme mit komplexem Behandlungsprogramm eine $\mathrm{HBO}$ erhielten und 23 Patienten, die ambulant eine HBO ohne begleitende andere Therapiemaßnahmen durchführten. Im Rahmen der HBO-Therapie atmeten die Patienten unter Druck nach einem festgelegten Behandlungsschema reinen Sauerstoff. Durch die Steigerung des Sauerstoffpartialdrucks in der Scala tympani der Perilymphe wird eine positive Beeinflussung der metabolisch gestörten Hörsinneszelle angenommen. Behandlungsschwerpunkte im Rahmen der Rehabilitationsmaßnahme waren aktive Krankengymnastik für die Halswirbelsäule kombiniert mit physikalischen Behandlungen, Entspannungstherapie und psychologische Betreuung zur Verbesserung der Krankheitsbewältigung. Alle Patienten hatten vor der Beantragung der HBOBehandlung leitliniengerecht bereits eine rheologische Behandlung oder Therapie mit Glukokortikoiden ohne den gewünschten Therapieerfolg erhalten.

Zum Zeitpunkt der Studie erfolgte die Stadieneinteilung der Tinnituspatienten entsprechend der Leitlinie von 1998. Folglich wurden Patienten mit akutem und subakutem Tinnitus in die Studie aufgenommen. 
Zur Erfassung von Angst und Depressivität wurde die deutsche Version der Hospital Anxiety and Depression Scale (HADS) eingesetzt. Für die differenzierte Erfassung von Fatigue wurde das Multidimensional Fatigue Inventory (MFI-20) verwendet. Die Lebensqualität wurde mit dem Fragebogen European Organization for Research and Treatment Quality of Life Questionnaire (EORTC QLQ-C30) erhoben. Die Patienten wurden zu drei Messzeitpunkten untersucht: am Beginn (t1) und am Ende (t2) der 2wöchigen Therapie sowie vier Wochen danach (t3). Alle 120 Patienten nahmen auch an der zweiten Fragebogenerhebung (t2) teil. An der dritten Fragebogenerhebung (t3) beteiligten sich 98 der 120 Patienten (81,7 Prozent). Als Kontrollgruppe diente eine Teilstichprobe aus der deutschen Allgemeinbevölkerung, welche im Jahr 1998 als eine bevölkerungsrepräsentative Stichprobe $(n=2037)$ ebenfalls mit den drei Fragebögen HADS, MFI und EORTC QLQ-C30 untersucht wurde.

Von den 120 Patienten waren 63 Prozent männlich, das Durchschnittsalter betrug 44,8 Jahre.

In allen Bereichen der gesundheitsbezogenen Lebensqualität haben die Patienten zu t1 signifikant ( $p<0,001$ ) ungünstigere Werte als die Allgemeinbevölkerung. Außerdem sind Fatigue, Angst und Depressivität in der Patientenstichprobe höher ausgeprägt. Bezüglich der gesundheitsbezogenen Lebensqualität (EORTC QLQC30) sind die Unterschiede zwischen Patienten und Allgemeinbevölkerung am stärksten in den Bereichen Finanzielle Schwierigkeiten $(d=1,84)$, Soziale Funktionsfähigkeit $(d=1,66)$, Kognitive Funktionsfähigkeit $(d=1,49)$, Schlafprobleme ( $d=1,43)$, Rollenfunktionsfähigkeit $(d=1,42)$ und Emotionale Funktionsfähigkeit $(d=$ 1,42) ausgeprägt (höchste Effektstärken). Hinsichtlich Fatigue dominiert die Skala Mentale Fatigue $(d=0,92)$, wogegen die Skalen Reduzierte Aktivität und Reduzierte Motivation geringere Einbußen aufweisen. Angst $(d=0,98)$ dominiert gegenüber der Depressivität $(d=0,53)$.

Die zu t1 erfassten HADS-Werte für Angst und Depressivität liegen in der gleichen Größenordnung wie die HADS Mittelwerte, welche in anderen Studien bei Tinnituspatienten ermittelt wurden.

Bezüglich der Verläufe der Lebensqualität bei den Patienten zeigt sich in allen EORTC-Skalen von t1 nach t2 eine Verbesserung. In der Folgezeit (Verlauf von t2 bis t3) bleiben die Werte in den meisten Skalen relativ konstant. Die meisten 
Verbesserungen finden sich in denjenigen Variablen, bei denen am Beginn die größten Defizite (größte Abstände zu den Werten der Allgemeinbevölkerung) zu verzeichnen sind. Vergleicht man die Werte am Untersuchungsende (t3) mit denen der Allgemeinbevölkerung, so bleiben noch immer deutliche Unterschiede bestehen, insbesondere in den Bereichen Finanzielle Schwierigkeiten, Rollenfunktionsfähigkeit, Soziale Funktionsfähigkeit, Kognitive Funktionsfähigkeit, Fatigue (EORTC QLQ-C30) und Schlafprobleme. Frauen weisen im Vergleich zu Männern in acht der 22 Variablen signifikant ungünstigere Werte zu t1 auf, insbesondere hinsichtlich Fatigue. Das Alter hat nur einen geringen Einfluss auf die Lebensqualität zu t1.

Tinnituspatienten sind bezüglich der gesundheitsbezogenen Lebensqualität in allen Bereichen signifikant eingeschränkt. Besonders ausgeprägt ist dies für die psychosozialen Bereiche. Im Laufe der Zeit bilden sich die Einschränkungen deutlich zurück. Auch andere Studien berichten von einer Verbesserung der gesundheitsbezogenen Lebensqualität nach Therapie. Da keine Kontrollgruppe ohne hyperbare Sauerstofftherapie untersucht wurde, kann hier nicht geklärt werden, welcher Teil der Verbesserung innerhalb der ersten zwei Wochen auf die HBO-Therapie und welcher auf Spontanremission oder auf andere Maßnahmen im Rahmen der Rehabilitation rückführbar ist. Damit soll die Studie in keiner Weise als Evaluationsinstrument dieser Therapie verstanden werden. Wesentlich ist, dass nach Untersuchungsende, also vier Wochen nach Ende der Sauerstofftherapie, noch immer erhebliche Abstände zur Allgemeinbevölkerung in der gesundheitsbezogenen Lebensqualität bestehen bleiben. Zwar gestatten die Ergebnisse wegen des Fehlens einer Kontrollgruppe keine Schlüsse über die Wirksamkeit der hyperbaren Sauerstofftherapie, sie zeigen aber das vielfältige Belastungsspektrum der Patienten (insbesondere im psychosozialen Bereich) auf.

Trotz der Einschränkung der Aussagekraft der Ergebnisse durch das Studiendesign zeigt die Arbeit, dass Tinnituspatienten hinsichtlich der gesundheitsbezogenen Lebensqualität stärker eingeschränkt sind als die Allgemeinbevölkerung und dass sich diese Einschränkungen im Zeitverlauf bessern. Die Abklärung hinsichtlich psychosozialer Belastungsfaktoren ist von großer Bedeutung, um notwendige unterstützende Therapieangebote bezüglich der bestehenden Einschränkungen anzubieten, mit dem Ziel das psychische Befinden und die gesundheitsbezogene Lebensqualität der Patienten zu verbessern. 


\section{Erklärung über die eigenständige Abfassung der Arbeit}

Hiermit erkläre ich, dass ich die vorliegende Arbeit selbstständig und ohne unzulässige Hilfe oder Benutzung anderer als der angegebenen Hilfsmittel angefertigt habe. Ich versichere, dass Dritte von mir weder unmittelbar noch mittelbar geldwerte Leistungen für Arbeiten erhalten haben, die im Zusammenhang mit dem Inhalt der vorgelegten Dissertation stehen, und dass die vorgelegte Arbeit weder im Inland noch im Ausland in gleicher oder ähnlicher Form einer anderen Prüfungsbehörde zum Zweck einer Promotion oder eines anderen Prüfungsverfahrens vorgelegt wurde. Alles aus anderen Quellen und von anderen Personen übernommene Material, das in der Arbeit verwendet wurde oder auf das direkt Bezug genommen wird, wurde als solches kenntlich gemacht. Insbesondere wurden alle Personen genannt, die direkt an der Entstehung der vorliegenden Arbeit beteiligt waren.

Datum

Unterschrift 



\section{Danksagung}

Nach Abschluss meiner Dissertation möchte ich allen herzlich danken, die mich bei der Durchführung und Fertigstellung dieser Arbeit unterstützt haben.

An erster Stelle möchte ich meinem leider viel zu früh verstorben primären Doktorvater Herrn Prof. Dr. med. R. Schwarz, ehemaliger Leiter der Selbstständigen Abteilung für Sozialmedizin des Instituts für Arbeits- und Sozialmedizin der Universität Leipzig, für die freundliche Überlassung des Themas und die stets hilfreiche und intensive Betreuung danken.

Ebenso danke ich Herrn Dr. med. V. Rust, ehemaliger Ärztlicher Direktor des Fachklinikums Brandis, der gemeinsam mit Herrn Prof. Dr. med. R. Schwarz die Anregung zu dieser Arbeit gab.

Ein besonderes Wort des Dankes möchte ich an meinen Doktorvater Herrn Prof. Dr. rer. nat. habil. A. Hinz, der die Fortführung der Betreuung der Dissertation übernommen hat, richten, ohne den ich niemals ein Licht am Ende der Doktorarbeit gesehen hätte. Er war mir zu jeder Zeit ein engagierter und geduldiger Doktorvater, dem ich für seine motivierende und professionelle Anleitung sowie für wertvolle Verbesserungsvorschläge zutiefst dankbar bin.

Ich danke Frau Dr. rer. med. A. Meyer, die mir stets Ansprechpartner war und mit Ihrem Wissen, Ihrer konstruktiven Kritik und Ihren vielen Ideen immer wieder den nötigen Anschwung gegeben hat.

Des Weiteren möchte ich mich bei meinen Eltern bedanken, ohne die ein Studium und eine Doktorarbeit niemals möglich geworden wären. 



\section{Lebenslauf}

\section{Angaben zur Person}

Name:

Geburtsdatum:

Geburtsort:

Familienstand:

Wohnort:

\section{Ausbildung}

2007

1996

1989-1996

1983-1996

1982-1983

1978-1982

1970-1978

\section{Berufstätigkeit}

01.09.1989-17.04.1996

Assistenzärztin für Innere Medizin

Beginn mit der Weiterbildung zur Fachärztin für Innere Medizin zunächst am Justizvollzugskrankenhaus

Februar 1990 Fortsetzung der Facharztausbildung entsprechend der Weiterbildungsordnung in der Medizinischen Klinik am Parkkrankenhaus Leipzig Dösen 
18.04.1996

19.04.1996-31.12.1997

31.12.1997

01.01.1998-31.08.1999

01.09.1999- 31.01. 2007

Seit Februar 2007

Seit 1994

\section{Weiterbildung}

1994

1998

2003

2004

2006

2004-2007
Oktober 1995 Fortsetzung der Facharztausbildung in der Rehabilitationsklinik Brandis, Arbeit als Stationsärztin und im Bereich internistische Funktionsdiagnostik

Abschluss der Facharztausbildung - Facharztprüfung mit Zuerkennung der Gebietsbezeichnung Innere Medizin

Weiterbeschäftigung als Fachärztin für Innere Medizin in der Rehabilitationsklinik Brandis

Kündigung wegen Konkurs der Klinik während der Zeit des Erziehungsurlaubes (seit 15.08.1997)

während der Zeit des Erziehungsurlaubes nebenberufliche Tätigkeit als Ärztin an der Klinik für Hyperbarmedizin Leipzig

Wiederaufnahme der Tätigkeit als Fachärztin für Innere Medizin am Fachklinikum Brandis

Oberärztin mit Schwerpunkttätigkeit Diabetologie am Heliosklinikum Borna, Klinik für Innere Medizin Aufgabengebiete: oberärztliche Leitung der Diabetesstation, Leitung des Diabeteszentrums, Mitarbeit im interdisziplinären Gefäßteam, Gefäßdoppler

selbstständige Tätigkeit als Notärztin im Rettungsdienst der Stadt Leipzig und im Landkreis Leipzig

Erlangung der Fachkunde Rettungsdienst, aktive Teilnahme als Notärztin im Rettungsdienst der Stadt Leipzig

Anerkennung der Zusatzbezeichnung Physikalische Therapie

Qualifikation als Tauchmediziner (GTÜM e.V.)

Anerkennung der Zusatzbezeichnung Sozialmedizin

Qualifikation als Druckkammerarzt/Hyperbarmediziner (GTÜM e.V.)

Belegung von Weiterbildungskursen zur Erlangung der Zusatzbezeichnung Diabetologie 



